\title{
Honey-Based Medicinal Formulations: A Critical Review
}

\author{
Md Lokman Hossain ${ }^{1}$ (D) , Lee Yong Lim ${ }^{1}$ (D), Katherine Hammer ${ }^{2}$, Dhanushka Hettiarachchi ${ }^{1}$ and \\ Cornelia Locher $1, *$ (i)
}

1 Division of Pharmacy, School of Allied Health, University of Western Australia, Crawley 6009, Australia; mdlokman.hossain@research.uwa.edu.au (M.L.H.); lee.lim@uwa.edu.au (L.Y.L.); dhanushka.hettiarachchi@outlook.com (D.H.)

2 School of Biomedical Sciences, University of Western Australia, Crawley 6009, Australia; katherine.hammer@uwa.edu.au

* Correspondence: connie.locher@uwa.edu.au

Citation: Hossain, M.L.; Lim, L.Y.; Hammer, K.; Hettiarachchi, D.;

Locher, C. Honey-Based Medicinal Formulations: A Critical Review. Appl. Sci. 2021, 11, 5159. https:// doi.org/10.3390/app11115159

Academic Editors: Daniel

Severus Dezmirean and Adela Ramona Moise

Received: 19 May 2021

Accepted: 30 May 2021

Published: 2 June 2021

Publisher's Note: MDPI stays neutral with regard to jurisdictional claims in published maps and institutional affiliations.

Copyright: (C) 2021 by the authors. Licensee MDPI, Basel, Switzerland. This article is an open access article distributed under the terms and conditions of the Creative Commons Attribution (CC BY) license (https:/ / creativecommons.org/licenses/by/ $4.0 /)$.

\begin{abstract}
Honey, a concentrated natural product, is produced by honeybees (Apis mellifera) from the nectar of flowers. It contains over 200 compounds that exert various biological or pharmacological activities, ranging from antioxidant, anti-inflammatory, antimicrobial, and antihypertensive to hypoglycemic effects. Due to the presence of a plethora of bioactive compounds, as well as unique physicochemical properties, honey has been widely used as medicine throughout human history along with its extensive utilization as common food and flavoring agent. The application of neat honey for therapeutic purpose, however, poses some difficulties such as the maintenance of a required therapeutic concentration over an adequate timeframe due to the problem of liquefaction and leakage. This has driven researchers to incorporate honey into a range of formulations, for example, hydrogels, dressings, ointments, pastes, or lozenges. After a brief discussion of the chemistry and medicinal use of honey, this review focuses on commercial honey-based medicinal formulations as well as in vitro, in vivo, and clinical studies on noncommercial honey formulations for the treatment of various ailments. In addition to this, it also covers the application of honey formulations and the evidence underpinning their use.
\end{abstract}

Keywords: honey; honey formulation; review

\section{Introduction}

\subsection{The Chemistry of Honey}

Honey is a natural sugary substance produced by a variety of honeybee species from the nectar of flowers or honeydew. Bees first collect nectar from flowering foliage before the collected nectar is treated in an internal pouch known as the crop where numerous enzymes break down sugars. The subsequent solution is disgorged by the bees into honeycombs within their hives. Lastly, liquid evaporation takes place by air currents generated by the fanning of bee wings [1]. The product, known as honey, is an extremely concentrated viscous solution composed of sugars (about 80\%), water (approximately 17\%) and 'other' constituents (approximately 3\%). Fructose and glucose tend to be the key honey sugars, with very minor quantities of other mono-, di-, or oligosaccharides also being present, for example, sucrose, maltose, isomaltose, nigerose, turanose, and maltulose [1]. Honey is acidic ( $\mathrm{pH} 3-5)$ in nature due to the breakdown of glucose into gluconic acid by glucose oxidase from the bee crop [1,2]. The water content in honey is a vital characteristic that influences its physicochemical and organoleptic properties such as color, taste, specific gravity, solubility, viscosity, and crystallization tendency, and it also plays a major role in the potential microbial contamination of honeys [2]. The smaller fraction (about 3\%) of 'other' constituents is considered to be very important in influencing not only the organoleptic characteristics of honeys but also the bioactivity profiles of various honeys. These 'other' honey constituents, which are often also referred to as its non-sugar constituents, 
comprise flavonoids (flavonols, flavones, flavanols, flavanones, anthocyanidin, chalcones, and isoflavones), phenolic and other organic acids, caroteinoid-like substances, proteins (enzymes), amino acids, minerals $\left(\mathrm{Ca}^{2+}, \mathrm{Cu}^{2+}, \mathrm{Fe}^{2+}, \mathrm{Mg}^{2+}, \mathrm{Mn}^{2+}, \mathrm{P}^{3+}, \mathrm{K}^{+}, \mathrm{Na}^{+}, \mathrm{Zn}^{2+}\right)$, vitamins (specifically vitamin $\mathrm{C}$, vitamin $\mathrm{B} 6$, thiamine, niacin, riboflavin, and pantothenic acid), pigments, and several other compounds. For instance, various enzymes, namely, invertase, amylase, catalase, and glucose oxidase, are expected to be found in honey, and proline is the major amino acid, contributing half of the total free amino acids [2]. The composition, color, and taste of honey, which is strongly linked to the presence of these non-sugar constituents, depends on its floral source, geographical origin, climatic factors, the bee species involved in honey production, human processing techniques, and the storage of the honey prior to its use [3].

\subsection{A Brief History of Honey Use}

The usage of honey is very well documented throughout history. For centuries, honey has been used as natural medicine for the management of a wide range of ailments, evident from its applications during the ancient Egyptian and Greek eras, in traditional Chinese, Indian, and Islamic medicines, and as a complementary medicine across many other countries. References to honey as medicine can be confirmed from ancient records, such as the Sumerian clay tablets (approximately 6200 BC), Egyptian papyri (1900-1250 BC), Veda (Hindu scripture), which is about 5000 years old, the Holy Qur'an, the Talmud, the Bible (in both the Old and the New Testaments), sacred books of India, China, Persia, and Egypt, and the writings of the ancient Greek physician Hippocrates (460-357 BC) [4,5]. Prehistoric Egyptians believed bees to be blessed and applied honey in a variety of medications such as dressings for wound healing [4,5]. Judeo-Christian/Islamic traditions believed honey to be a gift of God that refreshes both the mind and soul. In the Islamic holy Qur'an, there is a dedicated chapter describing the effort of bees in the formation of honey, and Hadith (saying of the Prophet Mohammad) concedes honey as a remedy for all illness. The ancient Greek physician Hippocrates recommended the use of honey-based mixtures for the management of different diseases such as fever, pain, and wounds. Chinese people used honey in numerous traditional remedies as found in Li Shizhen's 16th century medical writing 'Compendium of Materia Medica'. In more recent times, Russian and Chinese soldiers used honey for the healing of battle wounds. The usage of honey sustained into modern folk medicine, for example, for the treatment of coughs and sore throats, dry eye symptoms, leg ulcers, wounds, earache, gastric ulcers, and constipation [5]. Modern clinical research methods and molecular techniques have allowed for an in-depth understanding of honey as a medicine.

\subsection{Honey Bioactivities}

Honey shows protective effects in various physiological systems such as the cardiovascular, nervous, respiratory, and gastrointestinal system [5]. The beneficial role of honey is attributed to its high osmolarity, acidity, and ability to generate hydrogen peroxide and nitric oxide on contact with water, along with the presence of so-called non-peroxide factors like methylglyoxal (MGO), which, taken together, might exert antimicrobial and/or antioxidant activities [5]. In addition to this, phenolic compounds, organic acids, enzymes, nutrients, and other minor components also have potential anticancer, antiparasitic, antiviral, and antidiabetic activity [3,5].

Honey has been impregnated with other materials, for example, collagen, gelatin, starch, cellulose, alginate, or agarose, to derive medicinal formulations, which, compared to neat honey, might be more convenient to use and provide a target oriented therapeutic application. The US Food and Drug Administration (FDA) has approved several honey-based products. In the majority of these formulations, the incorporated honey is obtained from the tree genus Leptospermum, which is native to Australia and New Zealand, and it is commonly referred to as Manuka honey. These FDA-approved honey-based medicinal formulations consist of gels, dressings, ointments, and pastes. Review articles 
on honey have mainly described the chemistry and biological activity of honey [5-13]. Khachemoune et al., in 2011 [4] and in a more recent update in 2017 [14], discussed FDAapproved medical-grade honey products, focusing on the wound-healing potential of honey along with its mechanism of action. To date, however, no review has been undertaken of the academic literature on commercial honey-based formulations which are indicated for other disorders, beyond a sole focus on wound healing. This broader focus is adopted in this review, which discusses FDA-approved and nonapproved honey formulations alongside in vitro, in vivo, and clinical studies on honey-based medicinal formulations indicated for various ailments.

\section{Methodology}

The databases of PubMed, ScienceDirect, and Google Scholar were searched using different combinations of the following terms: 'honey formulation', 'honey as medicine', and 'honey research'. The headings and abstracts of the primary literature obtained from these searches were considered to select articles for comprehensive review, and the reference sections from each article were searched manually for additional publications of relevance to this review. The review starts with an overview of the main commercial suppliers of honey formulations, followed by a discussion of different types of formulations such as gels, dressings, syrups, ointments, creams, pastes, eye drops, and pastilles and the in vitro, in vivo, and clinical evidence available to support their medicinal use.

\section{Honey-Based Medicinal Formulations}

\subsection{Major Commercial Suppliers of Honey Medicinal Products}

Presently, there are several companies that supply or are developing honey products (mainly using Manuka honey) which are indicated for the treatment of dry eye symptoms, wounds, minor abrasions, lacerations, minor cuts, minor scalds and burns, diabetic foot ulcers, and burns. One of the leading manufacturers of honey medicinal products is the USA-based Derma Sciences, a company specializing in the development of formulations for tissue regeneration that manufacturers a number of Manuka honey products under the brand Medihoney ${ }^{\mathrm{TM}}$ (Table 1). The New Zealand-based companies, Manuka Health and ManukaMed, also manufacture several Manuka honey-based products. Other leading companies include the United Kingdom-based companies, Advancis Medical and Oswell Penda, Theo Manufacturing BV in the Netherlands, and another USA-based company, Links Medical Products Inc. Nature's Gold is an Australian company that manufactures a few honey-based formulations using Australian Manuka honey, and its therapeutic topical cream is the first honey-based product endorsed by the Australian Therapeutic Goods Administration (TGA).

Table 1. Commercial honey-based formulations.

\begin{tabular}{|c|c|c|c|c|c|c|c|}
\hline $\begin{array}{l}\text { Formulation } \\
\text { Type }\end{array}$ & $\begin{array}{c}\text { Commercial } \\
\text { Name }\end{array}$ & Manufacturer & $\begin{array}{l}\text { Type of } \\
\text { Honey }\end{array}$ & $\begin{array}{c}\text { Honey } \\
\text { Content }(\%)\end{array}$ & $\begin{array}{c}\text { Other } \\
\text { Ingredients }\end{array}$ & Claims Made on Treatments & Comments \\
\hline \multirow{4}{*}{ Gel } & $\begin{array}{l}\text { MediHoney } \\
\text { Wound Gel }\end{array}$ & Dermasciences & Leptospermum & 80 & $\begin{array}{l}15 \% \text { myristyl } \\
\text { myristate, } 5 \% \\
\text { Plantacare } 810\end{array}$ & $\begin{array}{l}\text { Diabetic foot ulcers, leg ulcers, } \\
\text { pressure ulcers/sores, 1st and 2nd } \\
\text { degree partial thickness burns, } \\
\text { donor sites, and traumatic and } \\
\text { surgical wounds }\end{array}$ & $\begin{array}{c}\text { FDA } \\
\text { approved }\end{array}$ \\
\hline & $\begin{array}{c}\text { Manuka Wound } \\
\text { Gel }\end{array}$ & Dermasciences & Leptospermum & 80 & $\begin{array}{l}\text { Hydrocolloids } \\
\text { and a natural oil }\end{array}$ & $\begin{array}{l}\text { Minor abrasions, lacerations, minor } \\
\text { cuts, minor scalds, burns, diabetic } \\
\text { foot ulcers, leg ulcers, pressure } \\
\text { ulcers/sores }\end{array}$ & $\begin{array}{c}\text { FDA } \\
\text { approved }\end{array}$ \\
\hline & Activon & $\begin{array}{l}\text { Advancis } \\
\text { Medical }\end{array}$ & Leptospermum & 100 & Nil & $\begin{array}{l}\text { Diabetic foot and leg ulcers, leg } \\
\text { ulcers, pressure ulcers/sores, 1st } \\
\text { and 2nd degree partial thickness } \\
\text { burns, grafted and donor sites, and } \\
\text { traumatic and surgical wounds, } \\
\text { minor burns, minor abraded skin }\end{array}$ & $\begin{array}{c}\text { FDA } \\
\text { approved }\end{array}$ \\
\hline & Revamil gel & Oswell Penda & $\begin{array}{l}\text { Revamil } \\
\text { source (RS) } \\
\text { honey }\end{array}$ & 100 & Nil & $\begin{array}{l}\text { Venous ulcers, cavity and tracking } \\
\text { wounds, infected, surgical and } \\
\text { chronic wounds, diabetic foot } \\
\text { ulcers, } 1 \text { st and } 2 \text { nd degree burns. }\end{array}$ & CE certified \\
\hline
\end{tabular}


Table 1. Cont

\begin{tabular}{|c|c|c|c|c|c|c|c|}
\hline $\begin{array}{l}\text { Formulation } \\
\text { Type }\end{array}$ & $\begin{array}{l}\text { Commercial } \\
\text { Name }\end{array}$ & Manufacturer & $\begin{array}{l}\text { Type of } \\
\text { Honey }\end{array}$ & $\begin{array}{c}\text { Honey } \\
\text { Content }(\%)\end{array}$ & $\begin{array}{c}\text { Other } \\
\text { Ingredients }\end{array}$ & Claims Made on Treatments & Comments \\
\hline & L-Mesitran Soft & $\begin{array}{l}\text { Theo } \\
\text { Manufacturing } \\
\text { BV }\end{array}$ & $\begin{array}{l}\text { Medical grade } \\
\text { honey }\end{array}$ & 40 & $\begin{array}{l}\text { hypoallergenic } \\
\text { lanolin, } \\
\text { propylene glycol, } \\
\text { PEG } 4000 \text {, and } \\
\text { vitamins C and E }\end{array}$ & $\begin{array}{l}\text { Light to moderately exuding } \\
\text { wounds, foot ulcers, leg ulcers, } \\
\text { pressure ulcers/sores, 1st and 2nd } \\
\text { degree partial thickness burns, } \\
\text { donor sites, and traumatic and } \\
\text { surgical wounds }\end{array}$ & $\begin{array}{c}\text { FDA } \\
\text { approved }\end{array}$ \\
\hline & ManukaPli Gel & Manuka Med & Leptospermum & 100 & Nil & $\begin{array}{l}\text { Leg ulcers, pressure ulcers, 1st and } \\
\text { 2nd degree burns (superficial and } \\
\text { partial-thickness), diabetic foot } \\
\text { ulcers, surgical wounds, and } \\
\text { traumatic wounds }\end{array}$ & $\begin{array}{c}\text { FDA } \\
\text { approved }\end{array}$ \\
\hline & $\begin{array}{l}\text { Manuka Aid } \\
\text { Wound Gel }\end{array}$ & Manuka Health & Leptospermum & 94 & Not mentioned & $\begin{array}{l}\text { Wounds including cuts, grazes, } \\
\text { abrasions, burns and skin ulcers }\end{array}$ & $\begin{array}{c}\text { FDA } \\
\text { approved }\end{array}$ \\
\hline & $\begin{array}{l}\text { Melladerm Plus } \\
\text { wound gel }\end{array}$ & SanoMed & $\begin{array}{l}\text { Honey from } \\
\text { Bulgaria }\end{array}$ & 45 & $\begin{array}{l}\text { PEG 4000, } \\
\text { propylene glycol } \\
\text { and glycerine }\end{array}$ & $\begin{array}{l}\text { Different types of wounds (minor } \\
\text { scalds, burns, diabetic foot ulcers, } \\
\text { leg ulcers, pressure ulcers/sores) }\end{array}$ & $\begin{array}{c}\text { FDA } \\
\text { approved }\end{array}$ \\
\hline & Medihoney & Dermasciences & Leptospermum & 80 & $20 \%$ plant waxes & $\begin{array}{l}\text { Diabetic foot ulcers, leg ulcers, } \\
\text { pressure ulcers/sores, 1st and } 2 \text { nd } \\
\text { degree partial thickness burns, } \\
\text { donor sites, and traumatic and } \\
\text { surgical wounds }\end{array}$ & $\begin{array}{c}\text { FDA } \\
\text { approved }\end{array}$ \\
\hline & $\begin{array}{c}\text { Manuka Wound } \\
\text { gel }\end{array}$ & Melcare & Leptospermum & 80 & $\begin{array}{l}\text { plant derived } \\
\text { hydrocolloids } \\
\text { and a natural oil }\end{array}$ & $\begin{array}{l}\text { Contact layer for wounds such as } \\
\text { venous ulcers, pressure ulcers } \\
\text { (I-IV), diabetic ulcers, 1st and 2nd } \\
\text { degree burns, surgical wounds, } \\
\text { donor and recipient graft sites, } \\
\text { sloughy, malodorous wounds and } \\
\text { also general first aid wound care }\end{array}$ & $\begin{array}{c}\text { FDA } \\
\text { approved }\end{array}$ \\
\hline & $\begin{array}{l}\text { Manuka Health } \\
\text { Wound Gel }\end{array}$ & Manuka Health & Leptospermum & $\begin{array}{l}\text { Not } \\
\text { mentioned }\end{array}$ & Gelling agent & $\begin{array}{l}\text { Abrasions, scrapes, minor cuts, } \\
\text { scalds, burns and surface wounds }\end{array}$ & $\begin{array}{c}\text { FDA } \\
\text { approved }\end{array}$ \\
\hline & SurgihoneyRO ${ }^{\mathrm{TM}}$ & $\begin{array}{l}\text { Matoke } \\
\text { Holdings and } \\
\text { SurgihoneyRO } \\
\text { Ltd. }\end{array}$ & Organic honey & $\begin{array}{l}\text { Not } \\
\text { mentioned }\end{array}$ & Not mentioned & $\begin{array}{l}\text { Wide range of acute and chronic } \\
\text { wound types, including leg ulcers, } \\
\text { pressure injuries, diabetic foot } \\
\text { ulcers, burns and surgical wounds }\end{array}$ & CE certified \\
\hline & $\begin{array}{l}\text { Paw Manuka } \\
\text { wound gel }\end{array}$ & Blackmores & Leptospermum & 80 & $\begin{array}{l}\text { Natural Gum, } \\
\text { bees wax, and } \\
\text { nourishing } \\
\text { organic oils }\end{array}$ & $\begin{array}{l}\text { Non-healing and chronic wounds, } \\
\text { traumatic, acute and surgical } \\
\text { wounds, malodorous and sloughy } \\
\text { wounds, burns and as a general } \\
\text { first aid }\end{array}$ & $\begin{array}{l}\text { Veterinary } \\
\text { product }\end{array}$ \\
\hline \multirow{6}{*}{ Dressing } & $\begin{array}{l}\text { Medihoney } \\
\text { Wound } \\
\text { Dressings }\end{array}$ & Dermasciences & Leptospermum & 95 & $\begin{array}{l}\text { 5\% Calcium } \\
\text { alginate }\end{array}$ & $\begin{array}{l}\text { Moderate to heavily exuding } \\
\text { wounds, diabetic foot ulcers, leg } \\
\text { ulcers, pressure ulcers/sores, 1st } \\
\text { and 2nd degree partial thickness } \\
\text { burns, donor sites, and traumatic } \\
\text { and surgical wounds }\end{array}$ & $\begin{array}{c}\text { FDA } \\
\text { approved }\end{array}$ \\
\hline & $\begin{array}{l}\text { Medihoney } \\
\text { Adhesive/Non- } \\
\text { Adhesive } \\
\text { Honeycolloid } \\
\text { Dressing }\end{array}$ & Dermasciences & Leptospermum & 80 & $\begin{array}{l}20 \% \text { sodium } \\
\text { alginate powder } \\
\text { (hydrocolloid } \\
\text { sheet) }\end{array}$ & $\begin{array}{l}\text { Diabetic foot ulcers, leg ulcers, } \\
\text { pressure ulcers/sores, 1st and 2nd } \\
\text { degree partial thickness burns, } \\
\text { donor sites, and traumatic and } \\
\text { surgical wounds }\end{array}$ & $\begin{array}{c}\text { FDA } \\
\text { approved }\end{array}$ \\
\hline & $\begin{array}{l}\text { Medihoney } \\
\text { dressing }\end{array}$ & Dermasciences & Leptospermum & 100 & Nil & $\begin{array}{l}\text { Diabetic foot ulcers, leg ulcers, } \\
\text { pressure ulcers/sores, first- and } \\
\text { second-degree partial-thickness } \\
\text { burns, donor sites, and traumatic } \\
\text { and surgical wounds }\end{array}$ & $\begin{array}{c}\text { FDA } \\
\text { approved }\end{array}$ \\
\hline & $\begin{array}{l}\text { Medihoney } \\
\text { Adhesive/Non- } \\
\text { Adhesive } \\
\text { Hydrogel } \\
\text { Colloidal Sheet }\end{array}$ & Dermasciences & Leptospermum & 63 & Adhesive border & $\begin{array}{l}\text { Non-draining to lightly exuding } \\
\text { wounds, diabetic foot ulcers, leg } \\
\text { ulcers, pressure ulcers/sores, 1st } \\
\text { and 2nd degree partial thickness } \\
\text { burns, donor sites, and traumatic } \\
\text { and surgical wounds }\end{array}$ & $\begin{array}{c}\text { FDA } \\
\text { approved }\end{array}$ \\
\hline & $\begin{array}{l}\text { Medihoney HCS } \\
\text { (Hydrogel } \\
\text { Colloidal Sheet) } \\
\text { Adhesive } \\
\text { dressing }\end{array}$ & Dermasciences & Leptospermum & $100 *$ & $\begin{array}{l}\text { Gelling agents } \\
\text { and colloidal } \\
\text { sheet }\end{array}$ & $\begin{array}{l}\text { Diabetic foot ulcers, leg ulcers, } \\
\text { pressure ulcers/sores, 1st and 2nd } \\
\text { degree partial thickness burns, } \\
\text { donor sites, and traumatic and } \\
\text { surgical wounds }\end{array}$ & $\begin{array}{c}\text { FDA } \\
\text { approved }\end{array}$ \\
\hline & $\begin{array}{l}\text { Manuka Foam } \\
\text { HC }\end{array}$ & $\begin{array}{l}\text { Links Medical } \\
\text { Products Inc. }\end{array}$ & Leptospermum & $100 *$ & $\begin{array}{l}\text { Absorbent } \\
\text { material }\end{array}$ & $\begin{array}{l}\text { Leg ulcers, pressure ulcers, 1st and } \\
\text { 2nd degree burns, diabetic foot } \\
\text { ulcers, surgical wounds, and } \\
\text { trauma wounds }\end{array}$ & $\begin{array}{c}\text { FDA } \\
\text { approved }\end{array}$ \\
\hline
\end{tabular}


Table 1. Cont.

\begin{tabular}{|c|c|c|c|c|c|c|c|}
\hline $\begin{array}{l}\text { Formulation } \\
\text { Type }\end{array}$ & $\begin{array}{l}\text { Commercial } \\
\text { Name }\end{array}$ & Manufacturer & $\begin{array}{l}\text { Type of } \\
\text { Honey }\end{array}$ & $\begin{array}{c}\text { Honey } \\
\text { Content }(\%)\end{array}$ & $\begin{array}{c}\text { Other } \\
\text { Ingredients }\end{array}$ & Claims Made on Treatments & Comments \\
\hline & Manuka Fill & $\begin{array}{l}\text { Links Medical } \\
\text { Products Inc. }\end{array}$ & Leptospermum & 100 & Nil & $\begin{array}{l}\text { Minor abrasions, lacerations, cuts, } \\
\text { and scalds; leg ulcers, pressure } \\
\text { ulcers, 1st and 2nd degree burns, } \\
\text { diabetic foot ulcers, surgical } \\
\text { wounds, and trauma wounds }\end{array}$ & $\begin{array}{c}\text { FDA } \\
\text { approved }\end{array}$ \\
\hline & $\begin{array}{l}\text { Manuka IG } \\
\text { wounds } \\
\text { dressings }\end{array}$ & $\begin{array}{l}\text { Links Medical } \\
\text { Products Inc. }\end{array}$ & Leptospermum & $100 *$ & $\begin{array}{l}\text { Carboxymethyl } \\
\text { cellulose (CMC) } \\
\text { as coating agent }\end{array}$ & $\begin{array}{l}\text { Minor abrasions, lacerations, cuts, } \\
\text { scalds and burns; leg ulcers, } \\
\text { pressure ulcers, diabetic foot ulcers, } \\
\text { surgical wounds, and traumatic } \\
\text { wounds }\end{array}$ & $\begin{array}{l}\text { FDA } \\
\text { approved }\end{array}$ \\
\hline & $\begin{array}{l}\text { Manuka IG Max } \\
\text { wounds dressing }\end{array}$ & $\begin{array}{l}\text { Links Medical } \\
\text { Products Inc. }\end{array}$ & Leptospermum & 100 & Nil & $\begin{array}{l}\text { Sterile, single-use dressings as } \\
\text { secondary dressings for heavily } \\
\text { exuding wounds, particularly for } \\
\text { treating deep wounds and cavities. }\end{array}$ & $\begin{array}{l}\text { FDA } \\
\text { approved }\end{array}$ \\
\hline & $\begin{array}{l}\text { MANUKAtex } \\
\text { wound dressings }\end{array}$ & ManukaMed & Leptospermum & $100 *$ & $\begin{array}{l}\text { Carboxymethyl } \\
\text { cellulose (CMC) } \\
\text { as coating agent }\end{array}$ & $\begin{array}{l}\text { Leg ulcers, pressure ulcers, first- } \\
\text { and second-degree burns, diabetic } \\
\text { foot ulcers, surgical wounds, and } \\
\text { traumatic wounds. }\end{array}$ & $\begin{array}{l}\text { FDA } \\
\text { approved }\end{array}$ \\
\hline & $\begin{array}{l}\text { MANUKAhd } \\
\text { wound dressings }\end{array}$ & ManukaMed & Leptospermum & $100 *$ & $\begin{array}{l}\text { Super absorbent } \\
\text { polymer (SAP) } \\
\text { fiber material }\end{array}$ & $\begin{array}{l}\text { Minor abrasions, lacerations, cuts, } \\
\text { scalds and burns; leg ulcers, } \\
\text { pressure ulcers, 1st and 2nd degree } \\
\text { burns, diabetic foot ulcers, surgical } \\
\text { wounds, and traumatic wounds }\end{array}$ & $\begin{array}{l}\text { FDA } \\
\text { approved }\end{array}$ \\
\hline & Activon Tube & $\begin{array}{l}\text { Advancis } \\
\text { Medical }\end{array}$ & Leptospermum & 100 & Nil & $\begin{array}{l}\text { Any wound, including slough, } \\
\text { necrotic and malodorous wounds, } \\
\text { pressure ulcers, leg ulcers, diabetic } \\
\text { ulcers, surgical wounds, burns, } \\
\text { graft sites, infected wounds, cavity } \\
\text { wounds and sinuses. }\end{array}$ & $\begin{array}{c}\text { FDA } \\
\text { approved }\end{array}$ \\
\hline & Activon Tulle & $\begin{array}{l}\text { Advancis } \\
\text { Medical }\end{array}$ & Leptospermum & 100 & Nil & $\begin{array}{l}\text { Minor wounds, ulcerations and } \\
\text { burns, abraded skin, and irritated } \\
\text { areas, diabetic foot ulcers, leg } \\
\text { ulcers, pressure ulcers/sores, 1st } \\
\text { and 2nd degree partial thickness } \\
\text { burns, grafted and donor sites, and } \\
\text { traumatic and surgical wounds }\end{array}$ & $\begin{array}{c}\text { FDA } \\
\text { approved }\end{array}$ \\
\hline & $\begin{array}{l}\text { Elasto-Gel } \\
\text { Manuka Honey } \\
\text { Wound dressing }\end{array}$ & $\begin{array}{l}\text { Southwest } \\
\text { Technologies }\end{array}$ & Leptospermum & $\begin{array}{l}\text { Not } \\
\text { mentioned }\end{array}$ & $\begin{array}{l}\text { sodium } \\
\text { polyacrylic acid, } \\
\text { glycerine, and } \\
\text { water }\end{array}$ & $\begin{array}{l}\text { Minor cuts and abrasions, scrapes, } \\
\text { surface wounds, minor scalds and } \\
\text { burns }\end{array}$ & $\begin{array}{l}\text { FDA } \\
\text { approved }\end{array}$ \\
\hline & Algivon & $\begin{array}{l}\text { Advancis } \\
\text { Medical }\end{array}$ & Leptospermum & $100 *$ & $\begin{array}{l}\text { Soft alginate } \\
\text { dressing } \\
\text { impregnated } \\
\text { with honey }\end{array}$ & $\begin{array}{l}\text { Any wound but especially pressure } \\
\text { ulcers, leg ulcers, diabetic ulcers, } \\
\text { surgical wounds, burns, graft sites, } \\
\text { infected wounds, cavity wounds } \\
\text { and sinuses. Ideal for cavities and } \\
\text { debriding and de-sloughing large } \\
\text { areas of necrotic and sloughy tissue. }\end{array}$ & $\begin{array}{c}\text { FDA } \\
\text { approved }\end{array}$ \\
\hline & Algivon Plus & $\begin{array}{l}\text { Advancis } \\
\text { Medical }\end{array}$ & Leptospermum & $100 *$ & $\begin{array}{l}\text { Reinforced } \\
\text { alginate dressing } \\
\text { impregnated } \\
\text { with honey }\end{array}$ & $\begin{array}{l}\text { Leg ulcers, pressure ulcers, diabetic } \\
\text { ulcers, fungating lesions, } \\
\text { complicated surgical wounds, } \\
\text { abrasions, infected wounds, cavity } \\
\text { wounds }\end{array}$ & $\begin{array}{c}\text { FDA } \\
\text { approved }\end{array}$ \\
\hline & $\begin{array}{l}\text { ManukaHD } \\
\text { LITE dressing }\end{array}$ & ManukaMed & Leptospermum & $100 *$ & $\begin{array}{l}\text { Honey is } \\
\text { impregnated } \\
\text { into a pad of } \\
\text { super absorbent } \\
\text { gelling fiber pad }\end{array}$ & $\begin{array}{l}\text { Leg ulcers, pressure ulcers, first- } \\
\text { and second-degree burns } \\
\text { (superficial- and partial-thickness), } \\
\text { diabetic foot ulcers, surgical } \\
\text { wounds, and traumatic wounds. }\end{array}$ & $\begin{array}{c}\text { FDA } \\
\text { approved }\end{array}$ \\
\hline & $\begin{array}{l}\text { ManukaHD } \\
\text { LITE ROPE } \\
\text { dressing }\end{array}$ & ManukaMed & Leptospermum & $100 *$ & $\begin{array}{l}\text { Honey is } \\
\text { impregnated } \\
\text { into a pad of } \\
\text { super absorbent } \\
\text { polymers }\end{array}$ & $\begin{array}{l}\text { Tunnelling and undermining } \\
\text { wounds, venous ulcers, diabetic } \\
\text { foot ulcers, press ulcers, and } \\
\text { surgical wounds. }\end{array}$ & $\begin{array}{c}\text { FDA } \\
\text { approved }\end{array}$ \\
\hline & Actilite & $\begin{array}{l}\text { Advancis } \\
\text { Medical }\end{array}$ & Leptospermum & 99 & 1\% Manuka oil & $\begin{array}{l}\text { Ulcers, leg ulcers, diabetic ulcers, } \\
\text { surgical wounds, burns, graft sites, } \\
\text { infected wounds, cavity wounds } \\
\text { and sinuses. }\end{array}$ & $\begin{array}{l}\text { FDA } \\
\text { approved }\end{array}$ \\
\hline & L-Mesitran Tulle & $\begin{array}{l}\text { Theo } \\
\text { Manufacturing } \\
\text { BV }\end{array}$ & $\begin{array}{l}\text { Medical grade } \\
\text { honey }\end{array}$ & 40 & $\begin{array}{l}\text { Polyethylene } \\
\text { dressing is } \\
\text { impregnated } \\
\text { with the patented } \\
\text { L-Mesitran } \\
\text { Soft gel }\end{array}$ & $\begin{array}{l}\text { Minor abrasions, lacerations, minor } \\
\text { cuts, and minor scalds and burns }\end{array}$ & $\begin{array}{c}\text { FDA } \\
\text { approved }\end{array}$ \\
\hline
\end{tabular}


Table 1. Cont.

\begin{tabular}{|c|c|c|c|c|c|c|c|}
\hline $\begin{array}{l}\text { Formulation } \\
\text { Type }\end{array}$ & $\begin{array}{l}\text { Commercial } \\
\text { Name }\end{array}$ & Manufacturer & $\begin{array}{l}\text { Type of } \\
\text { Honey }\end{array}$ & $\begin{array}{c}\text { Honey } \\
\text { Content }(\%)\end{array}$ & $\begin{array}{c}\text { Other } \\
\text { Ingredients }\end{array}$ & Claims Made on Treatments & Comments \\
\hline & $\begin{array}{l}\text { L-Mesitran } \\
\text { Hydro dressing }\end{array}$ & $\begin{array}{c}\text { Theo } \\
\text { Manufacturing } \\
\text { BV }\end{array}$ & $\begin{array}{l}\text { Medical grade } \\
\text { honey }\end{array}$ & 30 & $\begin{array}{l}\text { Acrylic polymer, } \\
\text { water and } \\
\text { polyurethane } \\
\text { film backing }\end{array}$ & $\begin{array}{l}\text { Low to moderate exuding wounds, } \\
\text { including chronic wounds like } \\
\text { pressure ulcers, venous and } \\
\text { diabetic ulcers, superficial and } \\
\text { acute wounds like cuts, abrasions } \\
\text { and donor sites, superficial and } \\
\text { partial thickness burns, and } \\
\text { fungating wounds. }\end{array}$ & $\begin{array}{c}\text { FDA } \\
\text { approved }\end{array}$ \\
\hline & $\begin{array}{l}\text { L-Mesitran } \\
\text { Border dressing }\end{array}$ & $\begin{array}{l}\text { Theo } \\
\text { Manufacturing } \\
\text { BV }\end{array}$ & $\begin{array}{l}\text { Medical grade } \\
\text { honey }\end{array}$ & 30 & $\begin{array}{l}\text { Acrylic polymer } \\
\text { gel, water, } \\
\text { polyurethane } \\
\text { film backing and } \\
\text { an adhesive } \\
\text { border }\end{array}$ & $\begin{array}{l}\text { Chronic wounds e.g pressure ulcers, } \\
\text { venous and diabetic ulcers, } \\
\text { superficial and acute wounds e.g., } \\
\text { cuts, abrasions and donor sites, } \\
\text { superficial and partial thickness } \\
\text { burns and fungating wounds. }\end{array}$ & $\begin{array}{c}\text { FDA } \\
\text { approved }\end{array}$ \\
\hline & $\begin{array}{l}\text { L-Mesitran Net } \\
\text { dressing }\end{array}$ & $\begin{array}{l}\text { Theo } \\
\text { Manufacturing } \\
\text { BV }\end{array}$ & Medical grade & 20 & $\begin{array}{l}\text { Acrylic polymer } \\
\text { gel, water and } \\
\text { polyester mesh }\end{array}$ & $\begin{array}{l}\text { Chronic wounds e.g., pressure } \\
\text { ulcers, venous and diabetic ulcers, } \\
\text { superficial and acute wounds e.g., } \\
\text { cuts, abrasions and donor sites, } \\
\text { superficial and partial thickness } \\
\text { burns and fungating wounds }\end{array}$ & $\begin{array}{c}\text { FDA } \\
\text { approved }\end{array}$ \\
\hline & MelMax dressing & Dermagenics & $\begin{array}{c}\text { Buckwheat } \\
\text { honey }\end{array}$ & $\begin{array}{c}\text { Not } \\
\text { mentioned }\end{array}$ & $\begin{array}{l}\text { Polyhydrated } \\
\text { ionogens } \\
\text { ointment }\end{array}$ & $\begin{array}{l}\text { Acute wounds: burns, surgical } \\
\text { wounds, traumatic wounds; } \\
\text { Chronic wounds: leg ulcers, } \\
\text { diabetic ulcers, decubitus ulcers }\end{array}$ & $\begin{array}{c}\text { FDA } \\
\text { approved }\end{array}$ \\
\hline & MelDra dressing & Dermagenics & $\begin{array}{c}\text { Buckwheat } \\
\text { honey }\end{array}$ & $\begin{array}{c}\text { Not } \\
\text { mentioned }\end{array}$ & Acetate fabric & $\begin{array}{l}\text { Acute wounds e.g., cuts, abrasions } \\
\text { and donor sites, superficial and } \\
\text { partial thickness burns and } \\
\text { fungating wounds; Chronic } \\
\text { wounds: leg ulcers, diabetic ulcers, } \\
\text { decubitus ulcers }\end{array}$ & $\begin{array}{c}\text { FDA } \\
\text { approved }\end{array}$ \\
\hline & $\begin{array}{l}\text { Manuka Health } \\
\text { Wound Dressing }\end{array}$ & Manuka Health & Leptospermum & $\begin{array}{l}\text { Not } \\
\text { mentioned }\end{array}$ & $\begin{array}{l}\text { Sheet of } \\
\text { hydrogel }\end{array}$ & $\begin{array}{l}\text { Abrasions, scrapes, minor cuts, } \\
\text { scalds, burns and surface wounds }\end{array}$ & $\begin{array}{c}\text { FDA } \\
\text { approved }\end{array}$ \\
\hline & HoneySoft & Taureon & $\begin{array}{l}\text { Chilean } \\
\text { multifloral } \\
\text { honey }\end{array}$ & $\begin{array}{l}\text { Not } \\
\text { mentioned }\end{array}$ & $\begin{array}{l}\text { Inert acetate } \\
\text { dressing } \\
\text { impregnated } \\
\text { with honey }\end{array}$ & $\begin{array}{l}\text { Wounds that require bacterial } \\
\text { control, e.g., ulcus cruris, diabetic } \\
\text { wounds, burns, acute and chronic } \\
\text { wounds, infected wounds, } \\
\text { oncologic wounds, and pressure } \\
\text { sores }\end{array}$ & $\begin{array}{c}\text { FDA } \\
\text { approved }\end{array}$ \\
\hline & $\begin{array}{l}\text { Manuka Health } \\
\text { Breast Pad }\end{array}$ & Manuka Health & Leptospermum & $\begin{array}{l}\text { Not } \\
\text { mentioned }\end{array}$ & Hydrogel sheet & $\begin{array}{l}\text { Designed to absorb milk leakage } \\
\text { whilst providing a cooling and } \\
\text { comforting sensation on contact. } \\
\text { The pliable gel conforms to the } \\
\text { breast while the flexible cloth } \\
\text { backing helps control movement } \\
\text { and prevent bra friction. }\end{array}$ & $\begin{array}{c}\text { FDA } \\
\text { approved }\end{array}$ \\
\hline & $\begin{array}{l}\text { Revamil wound } \\
\text { dressing }\end{array}$ & Oswell Penda & $\begin{array}{l}\text { Revamil } \\
\text { source (RS) } \\
\text { honey }\end{array}$ & 100 & Nil & $\begin{array}{l}\text { Ulcers, dry damaged skin, infected } \\
\text { and chronic wounds, burns and } \\
\text { blistering conditions }\end{array}$ & $\begin{array}{c}\text { FDA } \\
\text { approved }\end{array}$ \\
\hline & $\begin{array}{c}\text { Medihoney } \\
\text { Apinate dressing }\end{array}$ & Dermasciences & Leptospermum & $\begin{array}{l}\text { Not } \\
\text { mentioned }\end{array}$ & Calcium alginate & $\begin{array}{l}\text { Acute and chronic wounds: } \\
\text { leg/foot ulcers, pressure ulcers, } \\
\text { sloughy wounds, infected wounds, } \\
\text { malodorous wounds, donor and } \\
\text { recipient graft sites and burns and } \\
\text { surgical wounds. }\end{array}$ & $\begin{array}{c}\text { FDA } \\
\text { approved }\end{array}$ \\
\hline \multirow[b]{2}{*}{ Ointment } & $\begin{array}{l}\text { Revamil Honey } \\
\text { Balm wound } \\
\text { ointment }\end{array}$ & Oswell Penda & RS honey & 25 & Not mentioned & $\begin{array}{l}\text { Surface wounds, such as grazes and } \\
\text { wounds caused by the scratching of } \\
\text { eczema }\end{array}$ & CE certified \\
\hline & $\begin{array}{l}\text { L-Mesitran } \\
\text { Ointment }\end{array}$ & $\begin{array}{l}\text { Theo } \\
\text { Manufacturing } \\
\text { BV }\end{array}$ & $\begin{array}{l}\text { Medical grade } \\
\text { honey }\end{array}$ & 48 & $\begin{array}{c}\text { Hypoallergenic } \\
\text { lanolin, } \\
\text { sunflower oil, } \\
\text { cod liver oil, } \\
\text { Calendula } \\
\text { officinalis, Aloe } \\
\text { Barbadensis, } \\
\text { vitamin C \& E } \\
\text { and zinc oxide }\end{array}$ & $\begin{array}{l}\text { Pressure ulcers, venous and } \\
\text { diabetic ulcers, burns, fungating } \\
\text { wounds, superficial and acute } \\
\text { wounds e.g., cuts, abrasions and } \\
\text { donor sites. }\end{array}$ & $\begin{array}{c}\text { FDA } \\
\text { approved }\end{array}$ \\
\hline
\end{tabular}


Table 1. Cont.

\begin{tabular}{|c|c|c|c|c|c|c|c|}
\hline $\begin{array}{l}\text { Formulation } \\
\text { Type }\end{array}$ & $\begin{array}{l}\text { Commercial } \\
\text { Name }\end{array}$ & Manufacturer & $\begin{array}{l}\text { Type of } \\
\text { Honey }\end{array}$ & $\begin{array}{c}\text { Honey } \\
\text { Content }(\%)\end{array}$ & $\begin{array}{c}\text { Other } \\
\text { Ingredients }\end{array}$ & Claims Made on Treatments & Comments \\
\hline & $\begin{array}{l}\text { Actibalm } \\
\text { Manuka Honey } \\
\text { Lip Balm }\end{array}$ & $\begin{array}{l}\text { Advancis } \\
\text { Medical }\end{array}$ & Leptospermum & 100 & $\begin{array}{l}\text { White } \\
\text { pharmaceutical } \\
\text { grade petroleum } \\
\text { jelly }\end{array}$ & $\begin{array}{l}\text { The petroleum jelly in the lip balm } \\
\text { seals the skin preventing bacteria } \\
\text { from entering the affected area and } \\
\text { keeps the skin's moisture from } \\
\text { evaporating whilst the Manuka } \\
\text { honey antibacterial and healing } \\
\text { properties help heal and soothe and } \\
\text { replace moisture for dry }\end{array}$ & $\begin{array}{c}\text { FDA } \\
\text { approved }\end{array}$ \\
\hline & $\begin{array}{l}\text { Melcura Plus } \\
\text { Melladerm } \\
\text { Phyto } \\
\text { Honey-Based } \\
\text { Ointment }\end{array}$ & SanoMed & $\begin{array}{l}\text { Not } \\
\text { mentioned }\end{array}$ & 48 & $\begin{array}{l}\text { Anti-oxidant } \\
\text { agents }\end{array}$ & $\begin{array}{l}\text { Provides critical nutrients to } \\
\text { accelerate wound healing }\end{array}$ & $\begin{array}{c}\text { FDA } \\
\text { approved }\end{array}$ \\
\hline & $\begin{array}{l}\text { Manuka power } \\
\text { concentrated } \\
\text { ointment }\end{array}$ & Nature's Gold & Leptospermum & 30 & Not mentioned & $\begin{array}{l}\text { Calms and soothes red and } \\
\text { inflamed skin as well as protects the } \\
\text { skin dry, hot or windy days and } \\
\text { relieves the symptoms of chafing. } \\
\text { Assists with minor steam burns, } \\
\text { minor cuts and abrasions. } \\
\text { Moisturises and protects dry and } \\
\text { cracked lips. Lessens the sting and } \\
\text { itch of insect and mosquito bites }\end{array}$ & \\
\hline & $\begin{array}{l}\text { Manuka Power } \\
\text { Concentrated } \\
\text { Ointment }\end{array}$ & Nature's Gold & Leptospermum & 30 & $\begin{array}{l}\text { Sunflower seed } \\
\text { oil, Bees wax, } \\
\text { Coca seed butter, } \\
\text { Shea butte, } \\
\text { Lecithin }\end{array}$ & $\begin{array}{l}\text { Can be used on scratches, bites, } \\
\text { inflamed red skin, eczema, rashes } \\
\text { and anywhere the skin is damaged. } \\
\text { Helps to soothe and repair and } \\
\text { maintain healthy skin of pet. }\end{array}$ & $\begin{array}{l}\text { Veterinary } \\
\text { product }\end{array}$ \\
\hline \multirow{3}{*}{ Cream } & $\begin{array}{l}\text { Medihoney } \\
\text { Barrier Cream }\end{array}$ & Dermasciences & Leptospermum & 30 & $\begin{array}{l}\text { Purified water, } \\
\text { coconut oil, } \\
\text { German } \\
\text { chamomile } \\
\text { flower extract, } \\
\text { evening } \\
\text { primrose oil, aloe } \\
\text { vera, and } \\
\text { vitamin E }\end{array}$ & $\begin{array}{l}\text { Helps to protect the skin from } \\
\text { damage caused by friction or shear. } \\
\text { Helps prevent damage to skin } \\
\text { caused by frequent hand washing }\end{array}$ & $\begin{array}{l}\text { FDA } \\
\text { approved }\end{array}$ \\
\hline & $\begin{array}{l}\text { Aniwell-Manuka } \\
\text { Honey } \\
\text { Veterinary } \\
\text { Wound Cream }\end{array}$ & Aniwell & Leptospermum & 25 & Not mentioned & $\begin{array}{l}\text { Can easily and frequently be } \\
\text { applied to minor irritations of the } \\
\text { skin with its 'Stay-in-place' } \\
\text { formulation which won't warm up } \\
\text { and run off. }\end{array}$ & $\begin{array}{l}\text { Veterinary } \\
\text { product }\end{array}$ \\
\hline & $\begin{array}{l}\text { Therapeutic Skin } \\
\text { Cream with } \\
\text { Manuka Honey }\end{array}$ & Nature's Gold & Leptospermum & $\begin{array}{l}\text { Not } \\
\text { mentioned }\end{array}$ & Not mentioned & $\begin{array}{l}\text { Variety of common skin conditions } \\
\text { and complaints, including eczema, } \\
\text { dermatitis, sun and wind burn, } \\
\text { insect bites and stings, cracked } \\
\text { heels cuts and scratches, minor } \\
\text { burns and scalds, dry and cracked } \\
\text { skin, nappy rash }\end{array}$ & $\begin{array}{c}\text { TGA } \\
\text { approved }\end{array}$ \\
\hline Paste & Medihoney Paste & Dermasciences & Leptospermum & 100 & Nil & $\begin{array}{l}\text { Promotes a moisture-balanced } \\
\text { environment conducive to wound } \\
\text { healing }\end{array}$ & $\begin{array}{c}\text { FDA } \\
\text { approved }\end{array}$ \\
\hline \multirow{4}{*}{ Syrup } & ActiFlex Honey & Oregan & Leptospermum & $\begin{array}{l}\text { Not } \\
\text { mentioned }\end{array}$ & $\begin{array}{l}\text { Freeze-dried } \\
\text { bee-venom } \\
\text { powder and } \\
\text { Lucosamine }\end{array}$ & $\begin{array}{l}\text { Joint inflammation and arthritis } \\
\text { pain }\end{array}$ & \\
\hline & $\begin{array}{l}\text { Manuka honey } \\
\text { and Royal jelly } \\
\text { syrup }\end{array}$ & Oregan & Leptospermum & 80 & $\begin{array}{l}20 \% \text { freeze-dried } \\
\text { royal jelly } \\
\text { powder }\end{array}$ & $\begin{array}{l}\text { Stimulates physical performance } \\
\text { and general health improvement; } \\
\text { increases resistance to viral } \\
\text { infections }\end{array}$ & \\
\hline & $\begin{array}{l}\text { Manuka honey } \\
\text { and Proposli }\end{array}$ & Oregan & Leptospermum & 80 & $20 \%$ propolis & $\begin{array}{l}\text { Helps to boost immune system; } \\
\text { removes toxins and radionuclide } \\
\text { out of the body; regulates } \\
\text { metabolism, gastric and intestinal } \\
\text { function. }\end{array}$ & \\
\hline & Feropip Syrup & PIP d.o.o. & $\begin{array}{l}\text { Honeydew } \\
\text { honey }\end{array}$ & $\begin{array}{l}\text { Not } \\
\text { mentioned }\end{array}$ & $\begin{array}{l}\text { Aronia (Aronia } \\
\text { melanocarpa), } \\
\text { vitamin C, iron } \\
\text { (ferrous } \\
\text { fumarate), aqua, } \\
\text { citric acid and } \\
\text { strawberry } \\
\text { aroma }\end{array}$ & $\begin{array}{l}\text { Iron absorption is increased with } \\
\text { the addition of vitamin } C \text { and } \\
\text { honeydew honey, which is } \\
\text { exceptionally rich in minerals }\end{array}$ & \\
\hline
\end{tabular}


Table 1. Cont.

\begin{tabular}{|c|c|c|c|c|c|c|c|}
\hline $\begin{array}{l}\text { Formulation } \\
\text { Type }\end{array}$ & $\begin{array}{l}\text { Commercial } \\
\text { Name }\end{array}$ & Manufacturer & $\begin{array}{l}\text { Type of } \\
\text { Honey }\end{array}$ & $\begin{array}{c}\text { Honey } \\
\text { Content }(\%)\end{array}$ & $\begin{array}{c}\text { Other } \\
\text { Ingredients }\end{array}$ & Claims Made on Treatments & Comments \\
\hline & Fortepip Honey & PIP d.o.o. & Sage honey & $\begin{array}{c}\text { Not } \\
\text { mentioned }\end{array}$ & $\begin{array}{l}\text { Dry propolis } \\
\text { extract, alcohol } \\
\text { extract of spruce }\end{array}$ & $\begin{array}{l}\text { Coats the mucous membranes of } \\
\text { the pharynx, soothes throat } \\
\text { irritation and coughing, facilitates } \\
\text { breathing }\end{array}$ & \\
\hline Eye drop & $\begin{array}{l}\text { Optimel } \\
\text { Manuka+ Dry } \\
\text { Eye Drop }\end{array}$ & Melcare & Leptospermum & 98 & $\begin{array}{l}\text { Sodium } \\
\text { Chloride, } \\
\text { Benzoic Acid }\end{array}$ & $\begin{array}{l}\text { Suitable for people suffering with } \\
\text { medium to severe dry eye } \\
\text { symptoms }\end{array}$ & $\begin{array}{l}\text { Patent } \\
\text { pending }\end{array}$ \\
\hline \multirow{2}{*}{ Pastilles } & $\begin{array}{c}\text { Honey and } \\
\text { Propolis Pastille }\end{array}$ & Propolia & $\begin{array}{c}\text { Not } \\
\text { mentioned }\end{array}$ & 35 & $\begin{array}{l}8 \% \text { propolis } \\
\text { extract as well as } \\
\text { Gum Arabic and } \\
\text { agave syrup }\end{array}$ & Addressing respiratory issues & \\
\hline & $\begin{array}{l}\text { Manuka Honey } \\
\text { Pastilles }\end{array}$ & Larnac & Leptospermum & 90 & $\begin{array}{c}9.5 \% \\
\text { maltodextrine } \\
\text { and } 0.5 \% \\
\text { Calciumstearat }\end{array}$ & Not provided & \\
\hline
\end{tabular}

* Manufacturer's claim; given other ingredients are present, it is uncertain whether this refers to honey content or honey purity.

\subsection{Honey-Based Gels}

Honey can be formulated into gels/hydrogels by incorporating suitable gel-forming agent(s), with and without other supporting materials. FDA-approved commercial honey gels currently on the market (Table 1) typically contain the Leptospermum-derived Manuka honey. Honey is mixed with other agent(s) such as natural emollients, for example, myristyl myristate, plantacare, lanolin, propylene glycol, PEG-400, or different plant waxes. While only limited clinical trial data or other levels of evidence for the claims made for these specific commercial formulations are publicly available, the effectiveness of such gels can be derived from various in vitro [1,4,14-17] and in vivo studies [18-20], as well as from randomized clinical trials using noncommercial formulations, as summarized in Table 2 [21-23].

The effectiveness of Medihoney ${ }^{\mathrm{TM}}$ antibacterial wound gel has been demonstrated in published case reports $[24,25]$. When localized wound infection is present, the wound is placed into a state of persistent inflammation, which can become uncomfortable for the patient. Associated problems such as increased exudate and malodor can be difficult to manage with topical dressings and can be distressing for the patient, adversely affecting their quality of life. The Medihoney ${ }^{\mathrm{TM}}$ antibacterial gel is considered to be suitable for wound treatment and it assists with the management of all key symptoms generally found in wounds [24,25]. A study led by Bateman et al. demonstrated that Medihoney ${ }^{\mathrm{TM}}$ gel treatment significantly reduced wound size, pain, odor, and exudate, indicating that it reduced the bioburden of the wounds to enable them to progress toward healing [24]. A four-center feasibility study was undertaken by Dunford et al. to determine the effectiveness of Medihoney ${ }^{\mathrm{TM}}$ for the treatment of leg ulcers in terms of pain relief, odor control, and overall patient satisfaction. The findings of this study demonstrated that Medihoney ${ }^{\mathrm{TM}}$ gel decreased ulcer pain and size considerably and deodorized odorous wounds quickly [26].

Another widely used commercial product is Melcare's Manuka Wound Gel ${ }^{\mathrm{TM}}$ compris- $^{-}$ ing 80\% Manuka honey and a natural emollient (in the form of plant-derived hydrocolloids and a natural oil) that is indicated as a contact layer for wounds such as venous ulcers, pressure ulcers (I-IV), diabetic ulcers, first- and second-degree burns, surgical wounds, donor and recipient graft sites, sloughy, malodorous wounds, and general first aid wound care. A honey-based gel can also be prepared with $100 \%$ honey without the addition of other ingredients. Activon ${ }^{\mathrm{TM}}$ and ManukaPli ${ }^{\mathrm{TM}}$ gel, for instance, contain $100 \%$ medical grade Manuka honey sterilized by gamma radiation with no additives. On the other hand, Revamil Gel ${ }^{\mathrm{TM}}$, which is indicated for the management of a broad range of wounds, consists of $100 \%$ pure and sterilized Revamil Source (RS) honey. RS honey is produced by Bfactory Health Products (Rhenen, The Netherlands) under standardized conditions in greenhouses from healthy bee colonies living in a controlled environment. By utilizing this controlled production process, the company aims to provide reproducible wound healing 
and antibacterial activity in their Revamil honey gel products. The factors responsible for the antibacterial activity of this honey are its high sugar concentration, the generation of $\mathrm{H}_{2} \mathrm{O}_{2}$, the 1,2-dicarbonyl compound methylglyoxal (MGO), and the cationic antimicrobial peptide bee defensin-1, as well as its low $\mathrm{pH}$ (3.2) which is at the lower end of the $\mathrm{pH}$ range ( $\mathrm{pH}$ 3.2-4.5) found for honeys [27]. A low $\mathrm{pH}$ and high sugar content (common in all honeys) create an unfavorable environment for bacteria. A 2012 study by Kwakman et al. found that RS honey diluted in water to between $40 \%$ and $20 \%$ produced high levels of $\mathrm{H}_{2} \mathrm{O}_{2}$, with a maximum concentration of $5.62 \pm 0.54 \mathrm{mM} \mathrm{H} \mathrm{O}_{2}$ formed in $30 \%$ honey [28]. Conversely, MGO occurs at a relatively low concentration $(0.25 \mathrm{mM})$ compared to Manuka honey (up to $16.1 \mathrm{mM}$ ), although even this minor quantity of MGO contributes notably to the overall antimicrobial activity of RS honey. It has been demonstrated, however, that bee defensin-1 (a cationic antimicrobial peptide, AMP) and $\mathrm{H}_{2} \mathrm{O}_{2}$ are the key factors associated with the prompt bactericidal activity of RS honey [29]. Interestingly, another bee product, royal jelly, also contains bee defensin-1, which contributes to protection against microbial spoilage. In 2011, Kwakman et al. found that medical-grade honey enriched with AMP has greater activity against antibiotic-resistant pathogens. This effect is also seen in RS honey, which, when combined with BP2 (bactericidal peptide 2) rapidly kills bacteria and possesses a broader spectrum of bactericidal activity than either BP2 or RS honey alone [30]. In addition to human applications, honey-based gels (e.g., Paw Manuka gel ${ }^{\mathrm{TM}}$ ) can also be used as veterinary medicines, for example, for the treatment of wounds in dogs, cats, and horses.

\subsection{Honey-Based Dressings}

A variety of honey-based dressings are available in the market as over-the-counter (OTC) and prescription medicines. These dressings are indicated for different types of wounds, such as abrasions, lacerations, burns, traumatic and surgical wounds, diabetic foot ulcers, and leg ulcers. As can be seen from the information presented in Table 2, the evidence of the effectiveness of honey-based dressings can be derived from different in vitro and in vivo studies [31,32], as well as from clinical studies [33-36].

The honey used in these dressings is mainly New Zealand medical-grade Leptospermum Manuka honey, which may be mixed with other agents, such as polymers, purified water, lanolin, and various other natural agents. Buckwheat honey is another honey that has been formulated into medicinal dressings. Dermagenics manufactures MelMax ${ }^{\mathrm{TM}}$ wound dressing using buckwheat honey mixed with a polyhydrated ionogen ointment. DermaSciences manufactures honey-based dressings under the brand name 'Medihoney ${ }^{\mathrm{TM}}$ ', which comprise $95 \%$ active Manuka honey and 5\% calcium alginate. The alginate forms a gel by absorbing wound exudate, which helps to maintain a moist wound environment that aids the autolytic debridement [37]. Under the supervision of a healthcare professional, these Medihoney ${ }^{\mathrm{TM}}$ dressings may be prescribed to manage moderately to severely exuding wounds. Another product is the Medihoney Adhesive/Non-adhesive Honey Hydrocolloid Sheets ${ }^{\mathrm{TM}}$, which consist of $80 \%$ active Manuka honey and $20 \%$ sodium alginate, indicated for lightly to moderately exuding wounds. Similarly, hydrogel colloidal sheets with $63 \%$ Leptospermum honey with and without an adhesive border are directed for the management of non-draining to lightly exuding wounds. A study carried out by Johnson et al. showed that Medihoney ${ }^{\mathrm{TM}}$ may have a significant role to play in infection prophylaxis, as its findings confirmed that Medihoney ${ }^{\mathrm{TM}}$ is as effective as mupirocin in the prevention of catheter-associated infections [38]. A randomized controlled trial conducted by Robson et al. using topical Medihoney ${ }^{\mathrm{TM}}$ and a dressing (or dressing system) selected from Aintree University Hospitals NHS, Liverpool, UK in accordance with standard local practice found a $10 \%$ acceleration in the healing rate of honey-treated wounds compared to conventionally treated wounds [22]. Moreover, Biglari et al. undertook a prospective observational study using Medihoney ${ }^{\mathrm{TM}}$ focused explicitly on chronic pressure ulcers. They found that the honey-based formulation destroyed bacterial growth in all 20 ulcers treated, with $90 \%$ of patients showing complete wound healing after 4 weeks [39]. 
There are several other types of honey-based dressings available commercially. The New Zealand-based Manuka Health (Newmarket, Auckland) manufactures a glycerinbased honey-containing hydrogel sheet for wound covering, and another New Zealandbased company, ManukaMed Ltd. (Solway, Masterton), offers gauze-based, honeyimpregnated fiber pad wound coverings. United Kingdom-based Advancis Medical (Kirkby-in-Ashfield, Nottinghamshire) produces numerous types of Manuka honeyimpregnated coverings containing cellulose-based net dressings (Actilite ${ }^{\mathrm{TM}}$ and Activone Tulle $^{\mathrm{TM}}$ ) and alginate hydrogels (Algivon ${ }^{\mathrm{TM}}$, Algivon Plus ${ }^{\mathrm{TM}}$ ). Links Medical Products Inc. manufactures dressings like Manuka foam $\mathrm{HC}^{\mathrm{TM}}$, Manuka Fill ${ }^{\mathrm{TM}}$, Manuka $\mathrm{IG}^{\mathrm{TM}}$, and Manuka IG Max ${ }^{\mathrm{TM}}$, which are all composed of Leptospermum scoparium honey from New Zealand. The single-use Manuka foam $\mathrm{HC}^{\mathrm{TM}}$ consists of honey infused into an absorbent foam-fiber hybrid material, with a polyurethane border and either a silicone or acrylic adhesive. Manuka Fill ${ }^{\mathrm{TM}}$ is a sterile, single-use wound care dressing consisting of $100 \%$ Leptospermum scoparium honey from New Zealand wrapped in low-density polyethylene (LDPE) tubes following sterilization by gamma irradiation. Manuka $\mathrm{IG}^{\mathrm{TM}}$, a sterile, singleuse wound dressing, is formulated with honey infused into acetate gauze and coated with carboxymethyl cellulose (CMC). Medihoney ${ }^{\mathrm{TM}}$, Hydrogel Colloidal Sheets ${ }^{\mathrm{TM}}$, Manuka Foam $\mathrm{HC}^{\mathrm{TM}}$, Manuka Fill ${ }^{\mathrm{TM}}$, and Manuka $\mathrm{IG}^{\mathrm{TM}}$ are indicated for minor wounds (abrasion, lacerations, cuts, scalds, burns) when supplied as OTC products, but they can also be used for serious wounds (leg ulcers, pressure ulcers, first- and second-degree burns, diabetic foot ulcers, surgical wounds, and trauma wounds) under medical supervision.

MelMax ${ }^{\mathrm{TM}}$ and MelDra ${ }^{\mathrm{TM}}$ dressings are produced by Dermagenics in the USA. They contain buckwheat (Cliftonia monophylla) honey, which has been found to be rich in phenolic constituents. These constituents have been revealed to be responsible for higher anti-oxidant activity and modulation of $\mathrm{pH}$, triggering a cascade of regulatory processes which bring contaminated and chronic wounds back to suitable stages of healing [40,41]. MelMax $^{\mathrm{TM}}$ and MelDra ${ }^{\mathrm{TM}}$ dressings containing buckwheat honey are indicated for acute (burns, postoperative, and trauma) and chronic (ulcus cruris, diabetic, and pressure ulcers) wounds. Honey can also be formulated into a dressing for breastfeeding mothers. Manuka Health manufactures Breast Pads that are hydrogel pads impregnated with sterile medical-grade $\mathrm{MGO}^{\mathrm{TM}} 400+$ Manuka honey on a flexible cloth backing. These pads are designed to absorb milk leakage and, at the same time, provide a cooling and comforting feeling on contact. Major advantages of this formulation are claimed to be the pliability of the gel which provides comfortable contact with the breast skin, as well as its flexible cloth backing.

In addition to commercial honey-based dressing, information is available on in vitro and in vivo studies involving some noncommercial honey-containing dressings, for example, an unusual one containing Malaysia Gelam honey. A study conducted by Zohdi et al. found that the application of this honey hydrogel dressing significantly enhanced $(p<0.05)$ wound closure and accelerated the rate of re-epithelialization in Sprague-Dawley rats as compared to a control hydrogel and OpSite film dressing [32]. The dressings consisted of Gelam honey $(6 \%, 8 \%, 10 \%, 15 \%)$ mixed with 15\% polyvinyl pyrrolidone (PVP) (Kollidon 90), 1\% protein-free agar solution, and 1\% polyethylene glycol (PEG).

\subsection{Honey-Based Ointments}

Ointments help to entrap water, keep the skin moist, and provide an emollient protective film, which are all important elements for wound healing. L-Mesitran ${ }^{\mathrm{TM}}$ ointment, which is manufactured by Theo Manufacturing BV, Maastrich, the Netherlands, and can be used to treat a wide range of wounds, contains $48 \%$ medical-grade honey alongside other natural agents (medical grade hypoallergenic lanolin, sunflower oil, cod liver oil, vitamins C and E, and zinc oxide). Revamil Honey Balm Wound Ointment ${ }^{\mathrm{TM}}$ is manufactured by Oswell Penda and indicated for the prevention or treatment of infections, including those caused by multidrug-resistant bacteria. As mentioned earlier, Revamil source (RS) honey is a standardized medical-grade honey made under controlled environments in greenhouses. 
According to the manufacturer, Revamil ointment is mainly appropriate for the treatment of surface wounds, such as grazes and wounds caused by the scratching of eczema and for domestic first aid. One of the simplest honey ointments is Actibalm ${ }^{\mathrm{TM}}$ topical ointment, which is composed of only two ingredients, white pharmaceutical-grade petroleum jelly and Manuka honey. It aims to provide soothing relief for dry, cracked lips by creating a protective barrier to lock in moisture, shielding the skin to drying, and aiding in the restoration of dry skin. This product is, however, not suitable for application on broken skin or mucosal surfaces. Melcura Plus Melladerm Phyto ${ }^{\mathrm{TM}}$ is an ointment which contains $48 \%$ honey and antioxidant agents to provide a comprehensive wound care treatment option. With its honey content, the formulation aims to create an environment unfavurable for microorganism proliferation, remove devitalized tissue, reduce wound odor, and promote granulation and epithelialization. The Australian company Nature's Gold manufactures two ointments using Australian Manuka honey. Manuka Power Concentrated Ointment ${ }^{\mathrm{TM}}$ contains 30\% Manuka honey and claims to protect the skin on dry, hot, or windy days, lessen the sting and itch of insect and mosquito bites, relieve the symptoms of chafing, and to be beneficial in the treatment of minor cuts, minor steam burns, and abrasions. The company also produces Manuka Power Concentrated Ointment ${ }^{\mathrm{TM}}$ for pets prepared by blending 30\% Manuka honey with sunflower seed oil, bees wax, coca seed butter, shea butte, and lecithin. This ointment is recommended for treating scratches, bites, inflamed red skin, eczema, rashes, and otherwise damaged skin. The manufacturer also claims that it can be applied on skin folds and on paws to maintain a healthy pet skin.

\subsection{Honey-Based Creams}

To date, two commercial honey-based creams are available for human and animal use, both containing New Zealand Manuka honey. The clinical effectiveness of the Manuka honey-based creams is clearly evident from in vitro, in vivo [6,8,42,43], and clinical studies $[23,42,43]$. Medihoney Barrier Cream ${ }^{\mathrm{TM}}$ contains $30 \%$ Manuka honey that can be used on intact and at-risk skin, for example, on skin which might otherwise break due to incontinence. According to the manufacturer, the product helps to maintain skin moisture and $\mathrm{pH}$, and it is useful when applied around the wounds, under skin folds, and under incontinence pads where it acts as a defensive barrier to prevent skin breakdown. Aniwell Active Manuka Honey Vet (AMHVet) cream contains 25\% of 15+ UMF (an indication of its antibacterial strength) certified active NZ Manuka honey and is indicated for the treatment of wounds and a range of skin conditions (e.g., abrasion, lacerations, cuts, and burns) in all animals, both with and without dressings. Therapeutic Skin Cream ${ }^{\mathrm{TM}}$, manufactured by Nature's Gold, contains Australian Manuka honey and is approved by the Therapeutic Goods Administration in Australia. It claims to provide relief from a variety of common skin conditions and complaints, including eczema, dermatitis, sun and wind burn, insect bites and stings, cracked heels cuts and scratches, minor burns and scalds, dry and cracked skin, and nappy rash.

\subsection{Honey-Based Pastes}

Medihoney Paste ${ }^{\mathrm{TM}}$ is currently the only commercially available honey-based paste. It contains Leptospermum honey, but no further information on its composition is publicly available. According to the manufacturer, the paste is effective in the treatment of lightly to moderately exuding wounds, as well as donor sites and traumatic or surgical wounds. The formulation is claimed to possess numerous benefits such promoting a moist environment favorable for wound healing. Furthermore, the manufacturer also states that it is safe to use in tunneled wounds or wounds with undermining, and it aids the treatment of wounds with delayed healing by drawing fluid through the wound to the surface due to its osmotic potential. In doing so, the paste helps to liquefy nonviable tissue and to maintain more acidic $\mathrm{pH}$ levels (3.5-4.5) within the wound environment that can assist wound healing. 


\subsection{Honey-Based Syrups}

As part of what is known as apitherapy, honey has also been formulated as syrup together with other natural agents. The honeys used to date for preparing commercial syrups (Table 1) are Manuka, Honeydew, and Sage honey. ActiFlex ${ }^{\mathrm{TM}}$ honey syrup, for example, consists of pure Manuka honey, freeze-dried bee venom powder, and glucosamine with no artificial additives and coloring agents. The manufacturer claims that the formulation offers respite from joint inflammation and arthritis pain through the combined action of its active constituents. Although no direct verification of these claims is provided, the role of honey in the treatment of inflammation has been demonstrated in several in vitro studies [44-46]. Honey can also be mixed with other honeybee products such as royal jelly to formulate honey syrup as seen in a Manuka and Royal Jelly ${ }^{\mathrm{TM}}$ honey syrup, which contains $80 \%$ organic Manuka honey and 20\% freeze-dried royal jelly powder. This syrup claims to increase resistance to viral infections, restore physical performance, and improve general health. Oregan Royal Jelly Honey ${ }^{\mathrm{TM}}$ is produced with the help of a processing technology based on freeze-drying manufacturing which allows the physicochemical characteristics and biological activity of volatile royal jelly components to be maintained. Honey is also mixed with propolis, for example, Manuka honey (80\%) and propolis $(20 \%)$, to make syrup (Manuka and Propolis ${ }^{\mathrm{TM}}$ ). PIP is a company based in England, which manufactures Feropip $^{\mathrm{TM}}$ and Fortepip ${ }^{\mathrm{TM}}$. Feropip ${ }^{\mathrm{TM}}$ syrup contains Honeydew honey, concentrated juice of aronia (Aronia melanocarpa), vitamin C, iron (ferrous fumarate), citric acid, and strawberry aroma. It claims to be beneficial for the treatment of fatigue, tiredness, and exhaustion. Fortepip Honey Syrup ${ }^{\mathrm{TM}}$ contains sage honey, dry propolis extract, and alcohol extract of spruce, and it is marketed for the treatment of bronchitis and asthma. According to the manufacturer, it also soothes sore throat and vocal cords, as well as eases breathing.

\subsection{Honey-Based Eye Drops}

The only commercially available honey-based eye drop is 'Optimel Manuka+ Dry Eye Drops' (patent pending) manufactured by Melcare. It contains 98\% Leptospermum honey, sodium chloride, and benzoic acid. Benzoic acid is a commonly used food preservative that is quickly metabolized by the liver and is present naturally at high levels in honeys derived from various Leptospermum species. The manufacturer notes that benzoic acid is selected as the preferred preservative for this formulation because of its natural existence in honey, as well as its effectiveness in low $\mathrm{pH}$ solutions. Optimel eye drops are claimed to be suitable for chronic dry eye and blepharitis, as well as the treatment of sore, irritated eyes and eyelids by creating an environment that assists healing and stops additional damage. Upon application to the surface of the eye, the eye drops create a low $\mathrm{pH}$ environment which inhibits the growth of bacteria that otherwise may potentially colonize the traumatized surface. The overgrowth of ocular flora resulting from tear deficiency and meibomian gland disease can contribute to tear film instability and ocular surface damage [47]. Chronic lid disease can be treated with both topical and systemic antibiotics; however, with an increase in resistance among ocular pathogens, the efficacy of antibiotics has been considerably reduced [48]. The manufacturer claims that thrice daily applications of Optimel Manuka+ dry eye drops to the inferior conjunctival fornix of patients with tear deficiency and/or meibomian gland disease can notably reduce the colony-forming units (CFUs) in the eyelids after 1 month of treatment. The reduction in CFUs decreases the susceptibility to bacterial conjunctivitis and keratitis, as well as improves the clinical symptoms of dry eye disease, by decreasing the production of certain bacterial lipases that may hydrolyze the meibomian gland lipids [49]. In a similar vein, Albietz and Lenton demonstrated that the application of Leptospermum neat honey is associated with significant improvements in dry eye and meibomian gland dysfunction $[47,50]$. The benefits of a conjunctival application of honey (30-100\%) was also studied by Al-Waili using E. coli, Proteus sp., S. aureus, Klebsiella sp., and P. aeruginosa as bacterial species and adult Wistar Sprague-Dawley albino rats as experimental animals [51]. They found the treatment to 
be effective for bacterial conjunctivitis induced in rats by different human pathogens (i.e., E. coli, Proteus sp., S. aureus, Klebsiella sp., and P. aeruginosa) with no significant side-effects.

\subsection{Honey-Based Pastilles}

The French manufacturer Propolia offers honey- and propolis-based pastilles, consisting of $35 \%$ honey of a non-specified floral source and $8 \%$ propolis extract, as well as Gum Arabic and agave syrup, which are promoted broadly as addressing respiratory issue. Larnac Manuka Honey, on the other hand, uses active New Zealand Manuka honey in its honey pastille formulation, which contains $90 \%$ honey alongside maltodextrine $(9.5 \%)$ and calciumstearat $(0.5 \%)$.

\section{Discussion}

Most commercial and noncommercial honey-based medicinal formulations seem to be targeted at topical wound care applications with only a few products aiming for alternative routes of administration (e.g., syrups, eye drops, pastilles). All commercially available gels, dressings, ointments, creams, and pastes have common topical uses. They are, for example, indicated as OTC formulations for the treatment of minor abrasions, lacerations, minor cuts, minor scalds, and burns, whereas, under professional healthcare professional supervision, they are used for non-draining to moderately exuding wounds. They are also commonly intended for the management of diabetic foot ulcers, leg ulcers (venous stasis ulcers, arterial ulcers, and leg ulcers of mixed etiology), pressure ulcers/sores (partial and full thickness), first-degree and second-degree partial thickness burns, donor sites, and traumatic and surgical wounds.

Reviewing the literature on commercial and noncommercial honey formulations, it transpires that, to date, only a few different types of honey have been used in these preparations. As briefly outlined below, for most of these honeys, evidence of bioactivity supporting their use in these medicinal formulations is available. Leptospermum honey, also called Manuka honey, appears currently to be the honey used in a majority of the commercial formulations, most likely as it has been widely demonstrated to have high antioxidant and antibacterial activities, which are beneficial for most intended medicinal applications. Manuka honey contains methylglyoxal (MGO), which is derived from the precursor molecule dihydroxyacetone (DHA) present in the pollen and nectar that the bees collect from many Leptospermum species. Next to a low $\mathrm{pH}$ and high osmolarity (which is common to all honeys), MGO has been demonstrated to be a major contributor to the observed antibacterial activity of Leptospermum honey [6,8]. Different concentrations of Leptospermum honey have been used in commercial formulations ranging from $25 \%$ to $100 \%$ (Table 1). Some in vitro studies demonstrated promising activity for noncommercial formulations prepared with Leptospermum honey (13-80\%), for instance, antibacterial activity, thereby promoting wound healing (Table 2) $[27,52,53]$. Next to Manuka honey, Revamil source (RS) honey is a medical-grade honey that is presently approved for clinical application. The manufacturer withholds the details on the origin of this honey, stating only that it is produced by a standardized process in greenhouses which contributes to reproducible activity that is an important criterion for clinical application. [39]. The commercially available Revamil gel is, for instance, prepared from pure RS honey (Table 1). As mentioned previously, buckwheat honey, a dark honey high in antioxidant activity [54], has been formulated into dressing by Dermagenics in the USA under the brand name of $\mathrm{MelMax}^{\mathrm{TM}}$ and MelDra ${ }^{\mathrm{TM}}$. Researchers at Brock University revealed that buckwheat honey has strong antibacterial effects against antibiotic-resistance bacteria [54]. In a separate study comparing buckwheat honey with Manuka honey, scientists found that buckwheat honey contains a higher percentage of sugars, proteins, and total phenolics, as well as less MGO, and it exhibits equivalent antibacterial activity against the bacteria Staphylococcus aureus and Pseudomonas aeruginosa [55]. Like Manuka honey, Buckwheat honey contains many minerals that play an important role in human health and normal physiological functioning, although its concentrations of $\mathrm{Fe}, \mathrm{Mn}$, and $\mathrm{Zn}$ are even higher than those found in Manuka 
honey [55]. To what extent these minerals contribute to the honeys' bioactivities in a range of medicinal applications is, however, not documented in the literature. Kanuka honey is produced from the New Zealand Kanuka bush (Kunzea ericoides). It has negligible MGO levels but contains hydrogen peroxide. As demonstrated in in vitro studies, it shows antibacterial and antioxidant activity [55]. In a randomized controlled trial, it was also confirmed that $90 \%$ medical-grade Kanuka honey and $10 \%$ glycerin is an effective treatment approach for rosacea [56]. Sage honey, which has been used to prepare a syrup formulation, is made by bees from the nectar of Salvia officinalis or several other types of Salvia. Its high ratio of fructose to glucose sugars make it very slow to crystallize [57]. Sage honey is a good source of antioxidants, and it also contains high concentration of pre- and probiotics, which help in digestion and treating problems in the gastrointestinal tract. Only limited information is, however, available on the bioactivities of other honeys that have been incorporated into formulations, such as 'Honeydew honey' or 'Bulgarian honey'. Given the complexity of honey's phytochemical composition and associated bioactivities, which in many instances are directly related to the honey's floral and geographical origin, such generic descriptions of honeys are not helpful. In this light, claims of effectiveness in wound care made by manufacturers of such honeys need to be treated with caution. Gelam honey (GH) is a Malaysian monofloral honey which is somewhat different to the honeys discussed earlier as it is not produced by European honeybees but from nectar and pollen collected by Apis dorsata bees from Powell (Melaleuca cajupati), which is locally known as the 'Gelam tree'. The honey they produce is a popular Malaysian traditional medicine. Several in vitro and in vivo studies have revealed the effectiveness of Gelam honey in wound healing $[26,58,59]$.

It is interesting to note that the quality of information provided by manufacturers on the honey in their formulations varies greatly. While some producers stipulate the honey's floral origin, others do not disclose this information, which makes an assessment of the product and a comparison with other formulations less meaningful. Moreover, ingredients other than honey in these formulation also appear to be often not very well documented.

Although the reviewed honey formulations have mainly medicinal applications, their standardization, based on publicly available information, appears at best to be limited to stipulating the honey's floral origin and controlling the amount of honey used in the preparation. The exceptions are Manuka honey-based formulations, which often stipulate MGO levels in the preparation. As mentioned earlier, a honey's phytochemical composition, along with the range of its bioactivities, is strongly dependent on the honey's floral source and geographical origin, as well as time of harvest and handling. Ideally, as is commonly the case in quality phytomedicines, a honey-based formulation should, therefore, be standardized to its main bioactive constituent(s), which in turn would allow to better compare different formulations. Given the chemical complexity of honey and the relatively limited data available on the bioactive constituents of these honeys (beyond MGO levels in Leptospermum-derived honeys), unfortunately, such a level of quality control might currently be unattainable. However, honey-based formulations should at least be very particular in controlling the quality of the honey used, for instance, by clearly documenting its botanical source, year, and area of harvest. For most of the commercial honey-based formulations, these data are not publicly available and it is impossible to assess if the information is just not released or not collected in the first instance. However, for formulations assessed by in vitro, in vivo, and clinical studies, the honey used in the formulations should be well documented to allow for inter-study comparisons and correlations. In view of the information provided in Table 2, it is concerning that, for most of the studies published to date, this is not the case, with almost half of them not even providing information on the honey itself. The quality of these studies and the strength of their evidence must, therefore, be drawn into question. This should, however, not imply that past studies on honey-based formulations do not hold any value. To the contrary, the critical comments made above should be seen as an encouragement for more well-designed and well-documented studies 
in the field which will add to the growing body of evidence supporting the use of honey in a wide range of medicinal applications.

In future work, it might also be beneficial to expand the range of floral sources of honeys in these formulations, as honeys differ in their pharmacological effects and efficacies depending on their phytochemical characteristics. With growing research on a wide range of honeys and their bioactive constituents, a wider range of honeys should, therefore, be investigated for medicinal formulations. In a similar way, it might also be useful to expand the current range of formulation types. The majority of honey-based formulations to date have been for topical administration, with gels and dressings being most popular. Other applications (e.g., eye drops, syrups) have so far been rarely explored; the honey-based formulations for the treatment of a range of eye conditions appear to be an interesting first step to address this gap.

Table 2. Noncommercial honey-based formulations.

\begin{tabular}{|c|c|c|c|c|c|c|c|}
\hline $\begin{array}{c}\text { Formulation } \\
\text { Type }\end{array}$ & $\begin{array}{l}\text { Type of } \\
\text { Honey }\end{array}$ & $\begin{array}{c}\text { Honey } \\
\text { Percentage }\end{array}$ & $\begin{array}{c}\text { Other } \\
\text { Ingredients }\end{array}$ & $\begin{array}{l}\text { Level of } \\
\text { Evidence }\end{array}$ & Study & Findings & Reference \\
\hline \multirow{5}{*}{ Gel } & $\begin{array}{c}\text { Not } \\
\text { mentioned }\end{array}$ & 75 & $\begin{array}{l}\text { Chitosan, methyl } \\
\text { paraben, } \\
\text { triethanolamine, } \\
\text { purified water }\end{array}$ & $\begin{array}{l}\text { In vitro } \\
\text { In vivo }\end{array}$ & $\begin{array}{l}\text { Antibacterial activity was } \\
\text { determined by disc diffusion } \\
\text { assay using most common } \\
\text { burn bacterial infection. } \\
\text { The gel was tested for in vivo } \\
\text { burn healing using } \\
\text { burn-induced wounds in } 10 \\
\text { albino mice. Wound-healing } \\
\text { activity was calculated over a } \\
\text { period of } 9 \text { days by measuring } \\
\text { the burn diameter compared to } \\
\text { silver sulfadiazine. }\end{array}$ & $\begin{array}{l}\text { Modulation of wound } \\
\text { healing and } \\
\text { enhancement of tissue } \\
\text { regeneration and } \\
\text { recovery }\end{array}$ & [18] \\
\hline & $\begin{array}{c}\text { Not } \\
\text { mentioned }\end{array}$ & 35 & $\begin{array}{l}\text { Royal jelly, olive } \\
\text { oil-propolis } \\
\text { extract }\end{array}$ & In vivo & $\begin{array}{l}42 \text { Wistar male rats were used } \\
\text { in this study. Diabetes was } \\
\text { induced by intraperitoneal } \\
\text { injection of streptozotocin, and } \\
\text { skin of the upper dorsal part of } \\
\text { the rats was removed. }\end{array}$ & $\begin{array}{l}\text { Accelerated wound } \\
\text { repair in diabetic and } \\
\text { nondiabetic rats }\end{array}$ & [19] \\
\hline & $\begin{array}{c}\text { Not } \\
\text { mentioned }\end{array}$ & $1.5-3.5$ & $\begin{array}{c}\text { Polyvinyl } \\
\text { alcohol (PVA), } \\
\text { carboxymethy- } \\
\text { late chitosan, } \\
\text { (CM-Chitosan), } \\
\text { and water }\end{array}$ & In vivo & $\begin{array}{l}\text { Mice were anesthetized by } \\
\text { injection of xylazine and } \\
\text { ketamine, and then the shaved } \\
\text { dorsal fur of each animal was } \\
\text { wounded. }\end{array}$ & $\begin{array}{l}\text { Promotes wound } \\
\text { healing in mice. } \\
\text { Macroscopic wound } \\
\text { status was observed on } \\
\text { the } 3 \text { rd, } 7 \text { th, and } 10 \text { th } \\
\text { days after the wound } \\
\text { was inflicted. }\end{array}$ & [20] \\
\hline & $\begin{array}{c}\text { Not } \\
\text { mentioned }\end{array}$ & 70 & Alginate & In vivo & $\begin{array}{l}20 \text { male Wistar rats were } \\
\text { excised using dissecting } \\
\text { scissors and forceps. }\end{array}$ & $\begin{array}{l}\text { Promotes dermal } \\
\text { wound healing by the } \\
\text { regulation of healing } \\
\text { process. Wound healing } \\
\text { activity was observed } \\
\text { over a period of } 21 \\
\text { days. }\end{array}$ & [31] \\
\hline & $\begin{array}{l}\text { Kanuka honey } \\
\text { (Kunzea } \\
\text { ericoides) }\end{array}$ & 90 & $10 \%$ glycerin & RCT & $\begin{array}{l}\text { The gel was applied twice } \\
\text { daily for } 30-60 \text { min per } \\
\text { application, for } 8 \text { weeks in } 137 \\
\text { adults and observed for the } \\
\text { treatment of rosacea. }\end{array}$ & $\begin{array}{l}\text { Effective and } \\
\text { well-tolerated treatment } \\
\text { for rosacea }\end{array}$ & [56] \\
\hline Dressing & $\begin{array}{l}\text { Gelam honey } \\
\text { (Melaleuca } \\
\text { spp.) }\end{array}$ & $6,8,10,15$ & $\begin{array}{l}\text { 15\% polyvinyl } \\
\text { pyrrolidone } \\
\text { (PVP) (Kollidon } \\
\text { 90), } 1 \% \\
\text { protein-free agar } \\
\text { solution, and } 1 \% \\
\text { polyethylene } \\
\text { glycol (PEG) }\end{array}$ & In vivo & $\begin{array}{l}96 \text { male Sprague-Dawley rats } \\
\text { with burns created at specific } \\
\text { area by heated cylindrical } \\
\text { aluminum. }\end{array}$ & $\begin{array}{l}\text { Application of honey } \\
\text { hydrogel dressings } \\
\text { significantly enhanced } \\
(p<0.05) \text { wound closure } \\
\text { and accelerated the rate } \\
\text { of re-epithelialization as } \\
\text { compared to control } \\
\text { hydrogel and OpSite } \\
\text { film dressing. } \\
\text { Supported dermal } \\
\text { wound healing via } \\
\text { regulation of the } \\
\text { healing process }\end{array}$ & [32] \\
\hline
\end{tabular}


Table 2. Cont

\begin{tabular}{|c|c|c|c|c|c|c|c|}
\hline $\begin{array}{c}\text { Formulation } \\
\text { Type }\end{array}$ & $\begin{array}{l}\text { Type of } \\
\text { Honey }\end{array}$ & $\begin{array}{c}\text { Honey } \\
\text { Percentage }\end{array}$ & $\begin{array}{c}\text { Other } \\
\text { Ingredients }\end{array}$ & $\begin{array}{l}\text { Level of } \\
\text { Evidence }\end{array}$ & Study & Findings & Reference \\
\hline & Leptospermum & 80 & $\begin{array}{c}\text { Poly vinyl } \\
\text { alcohol (PVA) } \\
\text { and } 1 \% \text { borax }\end{array}$ & In vitro & $\begin{array}{l}\text { Antibacterial activity of } \\
\text { samples against } E \text {. coli and } S \text {. } \\
\text { aureus was assessed using a } \\
\text { viable cell count technique. } \\
\text { Cell proliferation analysis was } \\
\text { performed using human } \\
\text { fibroblast cells }\left(10^{4} \text { cells / well) }\right. \\
\text { that were seeded on the tested } \\
\text { sample and incubated for } 1 \\
\text { and } 3 \text { days. Each day, inverted } \\
\text { microscopic evaluation was } \\
\text { conducted to analyze cell } \\
\text { responses after incubation. }\end{array}$ & $\begin{array}{l}\text { Promotes wound } \\
\text { healing process }\end{array}$ & [33] \\
\hline & Leptospermum & 13 & $\begin{array}{l}2 \% \text { chitosan } \\
\text { concentration, } \\
15 \% \text { glycerol } \\
\text { concentration, } \\
\text { water, and } \\
\text { sodium } \\
\text { bicarbonate }\end{array}$ & In vitro & $\begin{array}{l}\text { Antibacterial activity of the } \\
\text { prepared dressing was } \\
\text { determined by agar diffusion } \\
\text { test against E. coli and S. aureus, } \\
\text { where activity was evaluated } \\
\text { using the size of the clear zone. }\end{array}$ & Antibacterial activity & [52] \\
\hline & Leptospermum & $\begin{array}{c}\text { Not } \\
\text { mentioned }\end{array}$ & $\begin{array}{l}\text { Chitosan and } \\
\text { gelatin }\end{array}$ & In vitro & $\begin{array}{l}\text { Antibacterial activity was } \\
\text { determined by the disc } \\
\text { diffusion method against } S \text {. } \\
\text { aureus, S. pyogenes, } A \text {. } \\
\text { baumannii, P. aeruginosa, and } \\
\text { P. mirabilis. }\end{array}$ & Antibacterial activity & [53] \\
\hline
\end{tabular}

\section{Conclusions}

This review covered a wide range of commercially available and experimental honeybased medicinal formulations. As discussed, several in vitro and in vivo studies to date have confirmed the effectiveness of honey in the treatment of various ailments. Moreover, the efficacy of honey has also been revealed in clinical studies through randomized controlled trials and case reports. Thus, there is strong evidence in support of the application of honey as a medicinal agent for the treatment of a variety of conditions. However, the review also revealed that there is an opportunity to further explore a wider range of honeybased formulations using a variety of honeys. Moreover, the physicochemical properties of honey-based formulations should be studied more extensively, along with collating more quality evidence to support any therapeutic claims made for these products. More attention should also be paid to standardization and the level of information provided for honey-based formulations.

With persistent technological advancement, information regarding honey's pharmacological action has augmented at a rapid pace. However, as discussed, there are still under-explored areas in the context of honey formulations, and it is hoped that this review article has contributed to a better understanding of the currently available honey-based formulations, highlighted gaps in current knowledge, and provided evidence-based support to continue to explore novel honey-based medicinal products.

Author Contributions: Conceptualization, M.L.H. and C.L.; methodology, M.L.H., C.L., and L.Y.L.; writing-original draft preparation, M.L.H.; writing—review and editing, C.L. and L.Y.L.; supervision, C.L., L.Y.L., K.H., and D.H.; project administration, C.L.; funding acquisition, C.L. All authors have read and agreed to the published version of the manuscript.

Funding: This research received no external funding.

Institutional Review Board Statement: Not applicable.

Informed Consent Statement: Not applicable.

Data Availability Statement: Not applicable.

Conflicts of Interest: The authors declare no conflict of interest. The funders had no role in the design of the study; in the collection, analyses, or interpretation of data; in the writing of the manuscript, or in the decision to publish the results. 


\section{References}

1. Minden-Birkenmaier, B.A.; Bowlin, G.L. Honey-Based Templates in Wound Healing and Tissue Engineering. Bioengineering 2018, 5, 46. [CrossRef]

2. Martinotti, S.; Ranzato, E. Honey, Wound Repair and Regenerative Medicine. J. Funct. Biomater. 2018, 9, 34. [CrossRef]

3. Cianciosi, D.; Forbes-Hernández, T.Y.; Afrin, S.; Gasparrini, M.; Reboredo-Rodriguez, P.; Manna, P.P.; Zhang, J.; Lamas, L.B.; Flórez, S.M.; Toyos, P.A.; et al. Phenolic Compounds in Honey and Their Associated Health Benefits: A Review. Molecules 2018, 23, 2322. [CrossRef]

4. Lee, D.S.; Sinno, S.; Khachemoune, A. Honey and Wound Healing. Am. J. Clin. Dermatol. 2011, 12, 181-190. [CrossRef]

5. Saranraj, P.; Sivasakthi, S.; Feliciano, G. Pharmacology of Honey: A Review. Biol. Res. 2016, 10, $271-289$.

6. Alvarez-Suarez, J.M.; Gasparrini, M.; Forbes-Hernandez, T.Y.; Mazzoni, L.; Giampieri, F. The Composition and Biological Activity of Honey: A Focus on Manuka Honey. Foods 2014, 3, 420-432. [CrossRef] [PubMed]

7. Cornara, L.; Biagi, M.; Xiao, J.; Burlando, B. Therapeutic Properties of Bioactive Compounds from Different Honeybee Products. Front. Pharm. 2017, 8, 412. [CrossRef] [PubMed]

8. Johnston, M.; McBride, M.; Dahiya, D.; Owusu-Apenten, R.; Nigam, P.S. Antibacterial activity of Manuka honey and its components: An overview. AIMS Microbiol. 2018, 4, 655-664. [CrossRef]

9. Libonatti, C.; Varela, S.; Basualdo, M. Antibacterial activity of honey: A review of honey around the world. J. Microbiol. Antimicrob. 2014, 6, 51-56. [CrossRef]

10. Oryan, A.; Alemzadeh, E.; Moshiri, A. Biological properties and therapeutic activities of honey in wound healing: A narrative review and meta-analysis. J. Tissue Viability 2016, 25, 98-118. [CrossRef]

11. Zulkhairi Amin, F.A.; Sabri, S.; Mohammad, S.M.; Ismail, M.; Chan, K.W.; Ismail, N.; Norhaizan, M.E.; Zawawi, N. Therapeutic Properties of Stingless Bee Honey in Comparison with European Bee Honey. Adv. Pharmacol. Sci. 2018, 2018, 6179596. [CrossRef]

12. Abd Jalil, M.A.; Kasmuri, A.R.; Hadi, H. Stingless Bee Honey, the Natural Wound Healer: A Review. Skin Pharm. Physiol. 2017, 30, 66-75. [CrossRef] [PubMed]

13. Abubakar, M.B.; Abdullah, W.Z.; Sulaiman, S.A.; Suen, A.B. A review of molecular mechanisms of the anti-leukemic effects of phenolic compounds in honey. Int. J. Mol. Sci. 2012, 13, 15054-15073. [CrossRef]

14. Saikaly, S.; Khachemoune, A. Honey and Wound Healing: An Update. Am. J. Clin. Dermatol. 2017, 18, 237-251. [CrossRef]

15. Cooke, J.; Dryden, M.; Patton, T.; Brennan, J.; Barrett, J. The antimicrobial activity of prototype modified honeys that generate reactive oxygen species (ROS) hydrogen peroxide. BMC Res. Notes 2015, 8, 20. [CrossRef]

16. Kassim, M.; Achoui, M.; Mustafa, M.R.; Mohd, M.A.; Yusoff, K.M. Ellagic acid, phenolic acids, and flavonoids in Malaysian honey extracts demonstrate in vitro anti-inflammatory activity. Nutr. Res. 2010, 30, 650-659. [CrossRef] [PubMed]

17. Mandal, M.D.; Mandal, S. Honey: Its medicinal property and antibacterial activity. Asian Pac. J. Trop. Biomed. 2011, 1, 154-160. [CrossRef]

18. El-Kased, R.F.; Amer, R.I.; Attia, D.; Elmazar, M.M. Honey-based hydrogel: In vitro and comparative in vivo evaluation for burn wound healing. Sci. Rep. 2017, 7, 9692. [CrossRef] [PubMed]

19. Rashidi, M.K.; Mirazi, N.; Hosseini, A. Effect of topical mixture of honey, royal jelly and olive oil-propolis extract on skin wound healing in diabetic rats. Wound Med. 2016, 12, 6-9. [CrossRef]

20. Afshari, M.J.; Sheikh, N.; Afarideh, H. PVA/CM-chitosan/honey hydrogels prepared by using the combined technique of irradiation followed by freeze-thawing. Radiat. Phys. Chem. 2015, 113, 28-35. [CrossRef]

21. Molan, P.C. The evidence and the rationale for the use of honey as wound dressing. Wound Pract. Res. J. Aust. Wound Manag. Assoc. 2011, 19, 204-220.

22. Robson, V.; Dodd, S.; Thomas, S. Standardized antibacterial honey (Medihoney) with standard therapy in wound care: Randomized clinical trial. J. Adv. Nurs. 2009, 65, 565-575. [CrossRef]

23. Gethin, G.; Cowman, S. Bacteriological changes in sloughy venous leg ulcers treated with manuka honey or hydrogel: An RCT. J. Wound Care 2008, 17, 241-247. [CrossRef]

24. Bateman, S.; Graham, T. The use of Medihoney ${ }^{\mathrm{TM}}$ Antibacterial Wound Gel on surgical wounds post-CABG. Wounds UK 2007, 3 , 76-83.

25. Simon, A.; Sofka, K.; Wiszniewsky, G.; Blaser, G.; Bode, U.; Fleischhack, G. Wound care with antibacterial honey (Medihoney) in pediatric hematology-oncology. Support Care Cancer 2006, 14, 91-97. [CrossRef]

26. Dunford, C.E.; Hanano, R. Acceptability to patients of a honey dressing for non-healing venous leg ulcers. J. Wound Care 2004, 13, 193-197. [CrossRef]

27. Kwakman, P.H.S.; Velde, A.A.T.; De Boer, L.; Speijer, D.; Vandenbroucke-Grauls, M.J.C.; Zaat, S.A.J. How honey kills bacteria. FASEB J. 2010, 24, 2576-2582. [CrossRef]

28. Kwakman, P.H.S.; Zaat, S.A.J. Antibacterial components of honey. IUBMB Life 2012, 64, 48-55. [CrossRef]

29. Kwakman, P.H.S.; Te Velde, A.A.; de Boer, L.; Vandenbroucke-Grauls, C.M.J.E.; Zaat, S.A.J. Two major medicinal honeys have different mechanisms of bactericidal activity. PLoS ONE 2011, 6, e17709. [CrossRef]

30. Kwakman, P.H.S.; de Boer, L.; Ruyter-Spira, C.P.; Creemers-Molenaar, T.; Helsper, J.P.F.G.; Vandenbroucke-Grauls, C.M.J.E.; Zaat, S.A.J.; te Velde, A.A. Medical-grade honey enriched with antimicrobial peptides has enhanced activity against antibiotic-resistant pathogens. Eur. J. Clin. Microbiol. Infect. Dis. 2011, 30, 251-257. [CrossRef] 
31. Nazeri, S.; Mirabzadeh Ardakani, E.; Babavalian, H.; Latifi, A.M. Evaluation of Effectiveness of Honey-Based Alginate Hydrogel on Wound Healing in Rat Model. J. Appl. Biotechnol. Rep. 2015, 2, $293-297$.

32. Mohd Zohdi, R.; Abu Bakar Zakaria, Z.; Yusof, N.; Mohamed Mustapha, N.; Abdullah, M.N. Gelam (Melaleuca spp.) Honey-Based Hydrogel as Burn Wound Dressing. Evid. Based Complement. Altern. Med. 2012, 2012, 843025. [CrossRef]

33. Tavakoli, J.; Tang, Y. Honey/PVA hybrid wound dressings with controlled release of antibiotics: Structural, physico-mechanical and in-vitro biomedical studies. Mater. Sci. Eng. C 2017, 77, 318-325. [CrossRef]

34. Zeleníková, R.; Vyhlídalová, D. Applying honey dressings to non-healing wounds in elderly persons receiving home care. J. Tissue Viability 2019, 28, 139-143. [CrossRef]

35. Subrahmanyam, M. Honey impregnated gauze versus polyurethane film (OpSiteR) in the treatment of burns-a prospective randomised study. Br. J. Plast. Surg. 1993, 46, 322-323. [CrossRef]

36. Moghazy, A.M.; Shams, M.E.; Adly, O.A.; Abbas, A.H.; El-Badawy, M.A.; Elsakka, D.M.; Hassan, S.A.; Abdelmohsen, W.S.; Ali, O.S.; Mohamed, B.A. The clinical and cost effectiveness of bee honey dressing in the treatment of diabetic foot ulcers. Diabetes Res. Clin. Pract. 2010, 89, 276-281. [CrossRef]

37. George, N.M.; Cutting, K.F. Antibacterial Honey (Medihoney): In-vitro Activity Against Clinical Isolates of MRSA, VRE, and Other Multi-resistant Gram-negative Organisms Including Pseudomonas aeruginosa. Wounds 2007, 19, $231-236$.

38. Johnson, D.W.; van Eps, C.; Mudge, D.W.; Wiggins, K.J.; Armstrong, K.; Hawley, C.M.; Campbell, S.B.; Isbel, N.M.; Nimmo, G.R.; Gibbs, H. Randomized, controlled trial of topical exit-site application of honey (Medihoney) versus mupirocin for the prevention of catheter-associated infections in hemodialysis patients. J. Am. Soc. Nephrol. 2005, 16, 1456-1462. [CrossRef]

39. Biglari, B.; vd Linden, P.H.; Simon, A.; Aytac, S.; Gerner, H.J.; Moghaddam, A. Use of Medihoney as a non-surgical therapy for chronic pressure ulcers in patients with spinal cord injury. Spinal Cord 2012, 50, 165-169. [CrossRef]

40. Van den Berg, A.J.; van den Worm, E.; van Ufford, H.C.; Halkes, S.B.; Hoekstra, M.J.; Beukelman, C.J. An in vitro examination of the antioxidant and anti-inflammatory properties of buckwheat honey. J. Wound Care 2008, 17, 172-178. [CrossRef]

41. Liang, Y.; Zhang, L.; Qu, Y.; Li, H.; Shi, B. Antibacterial activity of buckwheat honey added with ferrous lactate against Pseudomonas aeruginosa. LWT 2020, 117, 108624. [CrossRef]

42. White, R. Manuka honey in wound management: Greater than the sum of its parts? J. Wound Care 2016, 25, 539-543. [CrossRef]

43. Gethin, G.; Cowman, S. Case series of use of Manuka honey in leg ulceration. Int. Wound J. 2005, 2, 10-15. [CrossRef]

44. Tonks, A.J.; Cooper, R.A.; Jones, K.P.; Blair, S.; Parton, J.; Tonks, A. Honey stimulates inflammatory cytokine production from monocytes. Cytokine 2003, 21, 242-247. [CrossRef]

45. Timm, M.; Bartelt, S.; Hansen, E.W. Immunomodulatory effects of honey cannot be distinguished from endotoxin. Cytokine 2008, 42, 113-120. [CrossRef]

46. Tonks, A.J.; Dudley, E.; Porter, N.G.; Parton, J.; Brazier, J.; Smith, E.L.; Tonks, A. A 5.8-kDa component of manuka honey stimulates immune cells via TLR4. J. Leukoc. Biol. 2007, 82, 1147-1155. [CrossRef]

47. Albietz, J.M.; Lenton, L.M. Effect of antibacterial honey on the ocular flora in tear deficiency and meibomian gland disease. Cornea 2006, 25, 1012-1019. [CrossRef]

48. Cernak, M.; Majtanova, N.; Cernak, A.; Majtan, J. Honey prophylaxis reduces the risk of endophthalmitis during perioperative period of eye surgery. Phytother. Res. 2012, 26, 613-616. [CrossRef]

49. Molan, P.C. Why honey is effective as a medicine. 2. The scientific explanation of its effects. N. Z. Beekeep. 2001, 82, 22-40.

50. Mansour, A.M. Epithelial corneal oedema treated with honey. Clin. Exp. Ophthalmol. 2002, 30, 149-150. [CrossRef]

51. Al-Waili, N.S. Investigating the antimicrobial activity of natural honey and its effects on the pathogenic bacterial infections of surgical wounds and conjunctiva. J. Med. Food 2004, 7, 210-222. [CrossRef] [PubMed]

52. Sasikala, L.; Rathinamoorthy, R.; Dhurai, B. Optimization of process conditions for chitosan-manuka honey film as wound contact layer for wound dressings. Wound Med. 2018, 23, 11-21. [CrossRef]

53. Abd El-Malek, F.F.; Yousef, A.S.; El-Assar, S.A. Hydrogel film loaded with new formula from manuka honey for treatment of chronic wound infections. J. Glob. Antimicrob. Resist. 2017, 11, 171-176. [CrossRef]

54. Brudzynski, K.; Abubaker, K.; Wang, T. Powerful bacterial killing by buckwheat honeys is concentration-dependent, involves complete DNA degradation and requires hydrogen peroxide. Front. Microbiol. 2012, 3, 242. [CrossRef]

55. Deng, J.; Liu, R.; Lu, Q.; Hao, P.; Xu, A.; Zhang, J.; Tan, J. Biochemical properties, antibacterial and cellular antioxidant activities of buckwheat honey in comparison to manuka honey. Food Chem. 2018, 252, 243-249. [CrossRef] [PubMed]

56. Shaun Holt, S.; Braithwaite, I.; Hunt, A.; Riley, J.; Holliday, M.; Richard Beasley, R. A topical kanuka honey formulation is an effective treatment for rosacea. J. Am. Acad. Dermatol. 2015, 72 (Suppl. 1), AB37.

57. Tuberoso, C.I.G.; Jerković, I.; Bifulco, E.; Marijanovic, Z.; Congiu, F.; Bubalo, D. Riboflavin and lumichrome in Dalmatian sage honey and other unifloral honeys determined by LC-DAD technique. Food Chem. 2012, 135, 1985-1990. [CrossRef]

58. Yusof, A.M.; Ghafar, N.A.; Kamarudin, T.A.; Chua, K.-H.; Azmi, M.F.; Ng, S.-L.; Yusof, Y.A.M. Gelam honey promotes ex vivo corneal fibroblasts wound healing. Cytotechnology 2019, 71, 1121-1135. [CrossRef]

59. Hussein, S.Z.; Mohd Yusoff, K.; Makpol, S.; Mohd Yusof, Y.A. Gelam Honey Inhibits the Production of Proinflammatory, Mediators NO, PGE(2), TNF- $\alpha$, and IL-6 in Carrageenan-Induced Acute Paw Edema in Rats. Evid. Based Complement. Altern. Med. 2012, 2012, 109636. [CrossRef] 